\title{
RECENT ADVANCES IN BOLIDE ENTRY MODELING: A BOLIDE POTPOURRI*
}

\author{
D. O. REVELLE \\ Earth and Environmental Sciences Division, Los Alamos National Laboratory, Los Alamos, New \\ Mexico, 87545, USA \\ (E-mail: revelle@lanl.gov)
}

\begin{abstract}
In this paper, we will review recent research on numerous aspects of bolide entry into a planetary atmosphere, including such topics as the entry dynamics, energetics, ablation, deceleration, fragmentation, luminosity, mechanical wave generation processes, a total (panchromatic) power budget including differential and integral efficiencies versus time, etc. Fragmentation, triggered by stagnation pressures exceeding the bolide breaking strength, has been subsequently included in either a collective or non-collective wake behavior limit. We have also utilized the differential panchromatic luminous efficiency of ReVelle and Ceplecha (2002) to compute bolide luminosity. In addition we also introduce the concept of the differential and integral acoustic/infrasonic efficiency and generalized it to the case of mechanical wave efficiency including internal atmospheric gravity waves generated during entry. Unlike the other efficiencies which are assumed to be a constant multiple of the luminous efficiency, the acoustic efficiency is calculated independently using a "first principles" approach. All of these topics have been pursued using either a homogeneous or a porous meteoroid model with great success. As a direct result, porosity seems to be a rather good possibility for explaining anomalous meteoroid behavior in the atmosphere.
\end{abstract}

\section{Introduction and Overview}

This paper is an outgrowth of an invited talk with Dr. Zdenek Ceplecha of the Ondrejov Observatory in the Czech Republic and was presented at the Meteorids 2004 conference at the University of Western Ontario in London, Ontario, Canada. Since most of the second half of the material that was presented in London was on advances in bolide observational analyses that has already been published as a major paper in Meteoritics and Planetary Science (Ceplecha and ReVelle, 2005), we jointly decided that ReVelle would prepare all of the materials for the invited talk to be published in Earth, Moon and Planets.

There have been several recent advances in bolide entry modeling by ReVelle (2001a, b, c, d, e), in ReVelle and Ceplecha (2001f, g) and also in ReVelle (2002a, b), in ReVelle and Ceplecha (2002) and in ReVelle et al. (2004). These advances include a fragmentation and luminosity model of bolide entry based on an energetics end height approach, a detailed power

* Invited Paper Presented at Meteoroids 2004; Presented at University of Western Ontario, London, Ontario, Canada, August 16-20, 2004 
balance calibration during entry within a panchromatic pass-band and the concomitant evaluation of numerous differential as well as integral efficiencies for all major processes that are known to occur. In addition to the earlier proposed extremes of collective versus non-collective wake behavior, a new fragmentation mechanism involving the oscillation between collective wake and non-collective wake extremes has also now been proposed as well. We will systematically present all of these advances within this article.

\subsection{Hypersonic ENTRY BEHAVIOR AND METEORS AS INFRASONIC SOURCES}

Using conventional dimensional analysis more than a dozen dimensionless combinations of similarity parameters naturally arise for the analysis of hypersonic entry into a planetary atmosphere. Fortunately at any one time usually only about four to six of these appear to be important for any specific entry that we have already modeled. The expected aerodynamic characteristics include a large Reynolds number and a large Mach number in a hypersonic flow regime with strong shock waves and shock front radiation from a region of very high temperatures. These values are consistent with a rather small classical Knudsen number (continuum flow regime), but at high altitudes where the neutral gas mean free paths are quite large for smaller bodies, the opposite extreme of free molecular flow can also be evident. Additional numbers are discussed in Ceplecha et al. (1998).

The modern starting point for entry modeling is the classical single-body, stagnation point ablation, ballistic entry analysis. This is a wave drag dominated regime for a blunt body with negligible lift and thermal conduction into the interior. For large entry speeds compared to the escape velocity, line sources/modified line sources energy deposition occurs for sufficiently large bodies (see below with regard to the differential acoustic efficiency evaluation however). For a non-linear blast wave relaxation radius from a "small" source, small compared to the density scale height, infrasonic and more generally acoustic-gravity waves (AGW's) are radiated from the line source and these signals propagate to great ranges due to low atmospheric absorption effects. The blast radius is defined as the square root of the energy deposited per unit length along the trail divided by the ambient pressure at any height. This can also be expressed as the product of the Mach number and the bolide diameter with a numerical multiplying factor, $k$ (generally $k$ is $<5-10)$ due to fragmentation effects.

For blast radii $<\sim 10 \mathrm{~m}$, sound wave absorption by processes in the upper atmosphere is severe and infrasonic signals are very unlikely to be recorded at ground level. Bolides previously detected infrasonically range in blast radius from $10 \mathrm{~m}$ to $\sim 6 \mathrm{~km}$ (with the latter value indicative of the Tunguska bolide see below) and for corresponding source energies from $\sim 10^{-5} \mathrm{kt}$ to $\sim 10 \mathrm{Mt}$. 
The wavelengths of the infrasound generated at $x=10$ (at a radial distance $=10$ blast wave radii from the source) are $\sim 2.81$ blast wave radii and beyond $x=10$ the wave propagates as a weak shock disturbance while for downward propagation eventually decaying to a linear perturbation as an infrasonic wave. The waves radiated beyond $x=10$ are influenced by the refractive effects due to the instantaneous atmospheric sound and horizontal wind speed structure as defined at moderate ranges by Snell's Law of Acoustics. The line source blast wave analogy is precisely defined for a body traveling with $\mathrm{Ma} \gg 1$ and $d V / d t \equiv 0$, where $V$ is the instantaneous meteor velocity and the resulting energy deposition is within a cylinder. This results in a Mach cone whose half angle is exactly zero as if the body had infinite speed. In fact the predicted refraction can be quite sensitive to this Mach cone half angle if the entry angle is fairly steep, depending on the atmospheric structure at the time of entry.

\subsection{BOLIDE PROPERTIES}

The nominal properties of the various groups that have been previously identified are conveniently plotted below in Figure 1 in the form of a property diagram following group designations by Ceplecha et al. (1998) using a single-body model of homogeneous meteoroids with $\mu=2 / 3$.

In addition, as shown by ReVelle (1983, 2001e, 2002a, b), there is also the question of whether some of the extreme end height behavior witnessed in the atmosphere is due to the fact that the entering particles are moderately or even highly porous (such as the Tagish Lake meteoroid/meteorites for example). This end height behavior is considered to be extreme in the sense that a $30 \mathrm{~km}$ end height difference is readily apparent for bodies whose parameters are otherwise identical (mass, velocity, shape, entry angle, etc.) with the exception of the statistical bolide group designation. This progressive increase in end height behavior increases steadily for Group I (ordinary chondrites), to Group II (carbonaceous chondrites) to Group IIIA and finally to group IIIB (strong and weak cometary materials respectively). The uniform bulk porosity values originally inferred by ReVelle (1983, 2001e, $2002 b)$ directly from the U.S.A. Prairie Network flight data range from $50 \%$ (for Group II), 75\% (for Group IIIA) to $91 \%$ (for Group IIIB). Since numerous spectra of the meteor showers indicates that the "toughest" Geminids are virtually identical in composition to the very weakest "Draconids", then a very natural way of describing these bodies is through their degree of porosity. We have also examined the form of this property diagram for porous bodies and have found that it is extremely similar to the results indicated in Figure 1. 


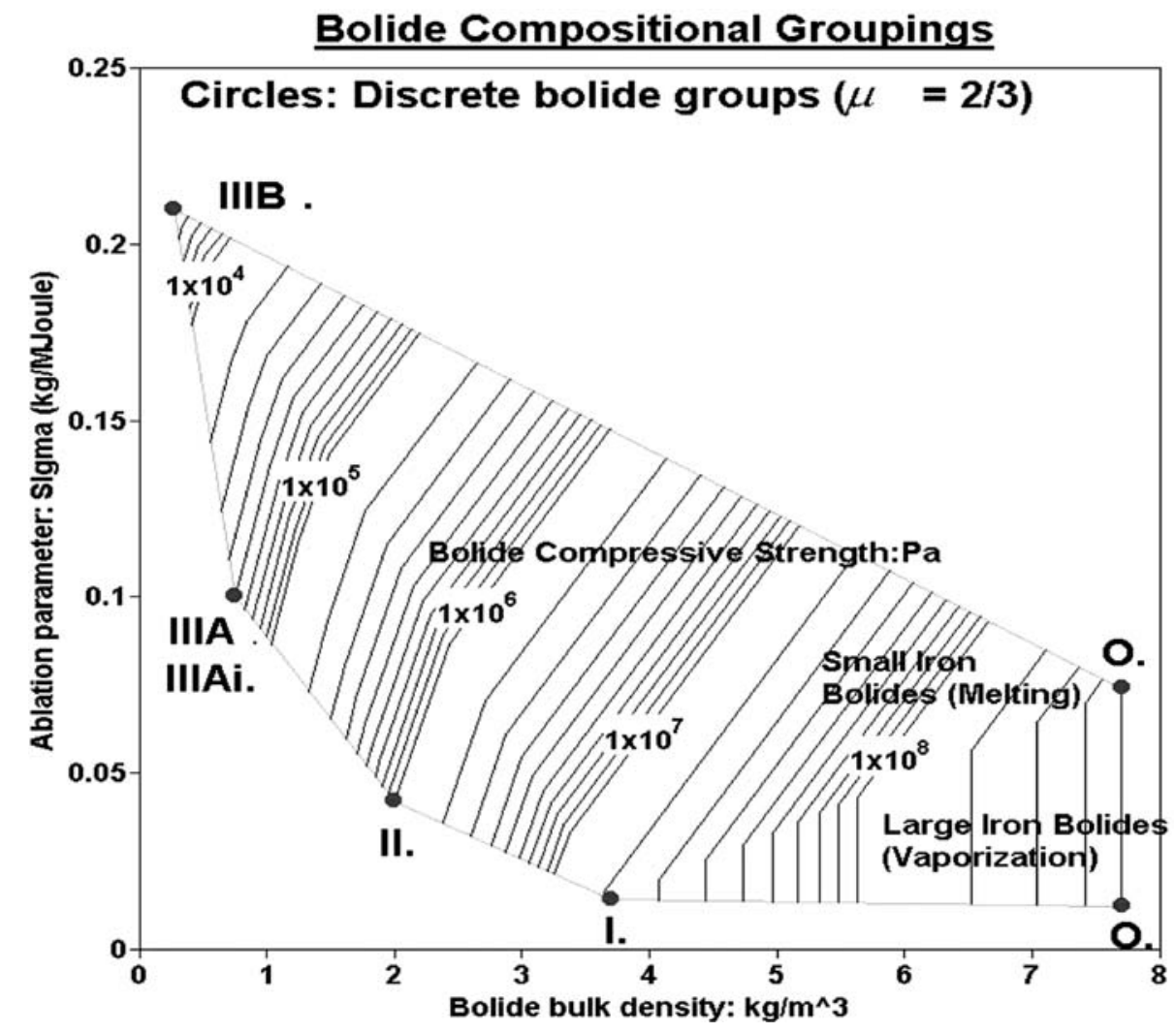

Figure 1. Bolide property diagram, i.e., values of the bulk density $\left(\mathrm{kg} / \mathrm{m}^{3}\right)$ versus the mean ablation parameter $(\mathrm{kg} / \mathrm{MJ})$ during entry as a function of contoured values of the breaking strength $(\mathrm{Pa})$ for meteoroids which were assumed to be homogeneous in their density.

As is discussed in detail subsequently below, an extended version of our original single-body, radiative-hydrodynamic entry model simulation ( $\mathrm{ReV}$ elle, 1979) has been modified into an energetics format and is shown to be equivalent to the classical drag dominated, constant sigma solution approach derived in the Appendix A (using the appropriate $D$ parameter for the specified level of kinetic energy remaining at the end height). This new energetics model was subsequently used along with a triggered progressive fragmentation model (with stagnation pressure as the mechanical triggering mechanism) and a panchromatic luminous emission model as described in this paper and in ReVelle (2001a-2001e) and ReVelle (2002a). In this work the ablation parameter is fully a function of height and of the corresponding classical Knudsen number for the case of local heat transfer to the body (for more details see below). 


\section{Mathematical/Physical Entry Model}

\subsection{AtMosPheric MODEL TYPeS}

In our modeling we have systematically investigated four specific entry cases, namely a constant or a height variable ablation parameter solution (using the $\sigma$ parameter) with either a hydrostatic, isothermal model or a fully non-isothermal, hydrostatic model atmosphere that reproduces the U.S. Standard Atmosphere series in middle latitudes in summer and in winter. Input variables include surface values $(z=0)$ of the air temperature, sound speed, mean free path, air pressure and then air density can be calculated from the ideal gas law.

These separate cases are all linked to the full physics solutions including ablation, deceleration, fragmentation and panchromatic luminosity, etc. for either a homogeneous or a porous meteoroid model. Atmospheric density and pressure wave variability in the form of turbulence and upward propagating internal gravity waves (IGW) from tropospheric sources are also possible to represent in such models, but will not be reported on here.

It should be noted that there is also a distinct limitation of this approach as applied to other planetary atmospheres. This fundamental limitation is directly due to the fact that the radiative heat transfer coefficient part of the ablation coefficient, $\sigma$, has been computed for the typical mean composition, i.e., primarily $\mathrm{N}_{2}$ and $\mathrm{O}_{2}$ at altitudes below $100 \mathrm{~km}$, for Earth's atmosphere. A separate radiation code computation would first be required to determine entry values for a large number of bolide radii, geopotential altitudes and velocities so that the results could be curve fit in a manner similar to that in Revelle (1979).

\subsection{Single-body approximation: ENERGetics formulation}

Using standard notation (except as otherwise defined), the end height (where the luminous trajectory ceases) for the case of a single-body is expressed below in the energetics form of ReVelle $(1981,1987,1993)$. It is demonstrated in Appendix A that this equation is equivalent to the standard end height equation (ReVelle, 1979). The $D$ parameter of ReVelle expresses the degree to which the original kinetic energy of the body has been removed at the predicted end height with $D=4.605$ corresponding to $99 \%$ energy removal and $D=2.303$ corresponding to $90 \%$ kinetic energy removal, etc.

\subsubsection{Energetics formulation for the end height: single-body model}

$$
z_{\mathrm{KE}}(V)=-H_{p} \cdot\left\{\ln \left(p_{\infty}^{*} / p_{0}\right) \cdot \exp \left[-(\sigma \cdot F) \cdot V_{\infty}^{2}\right] \cdot\left\{D-D^{\prime}\right\}+\exp \left[z^{\prime} / H_{p}\right]\right\}
$$




$$
\begin{aligned}
z^{\prime}= & -H_{p} \cdot\left\{\ln \left(p_{\infty}^{*} / p_{0}\right) \cdot\left(2 g H_{p} / V_{\infty}^{2}\right)\right\}=\text { Upper boundary condition } \\
D^{\prime}= & -\left\{E i\left[(\sigma \cdot F) \cdot V_{\infty}^{2}\right]-E i\left[(\sigma \cdot F) \cdot V^{2}(z)\right]\right. \\
& \left.-\ln \left(V_{\infty} / V(z)\right)^{2}-\left[(\sigma / 2)\left(V_{\infty}^{2}-V^{2}(z)\right)\right]\right\}
\end{aligned}
$$

where $\operatorname{Ei}(\sigma \cdot F)=$ the exponential integral function; $F=(1-\mu) / 2=$ constant; $\sigma=$ ablation coefficient as a function of height; $-2(*) \leq \mu \leq \sim 1 ; \mu=2 / 3=$ selfsimilar value (no shape change); $p_{\infty}{ }^{*}=\mathrm{mg} \cdot \sin \theta /\left(C_{D} A\right)=$ modified ballistic entry parameter; $m=$ instantaneous meteor mass $\theta=$ horizontal entry angle ( $>\sim 5-10$ degrees using Cartesian coordinates); $p_{\infty}^{*}=4 \cdot \rho_{m} \cdot r \cdot g \cdot \sin \theta /\left(3 C_{D}\right)$ for a sphere; $p_{0}=$ surface pressure; $r=$ instantaneous meteor radius; $D=4.605$ for $99 \%$ kinetic energy depletion at the end height

\section{$(*)$ : Effective "pancake" model limits for $\mu<0$}

As discussed in ReVelle (2001a, 2002a), the distinction between the singlebody regime and the catastrophic fragmentation limit can be separated on the basis of the ratio of the pressure scale height and the new quantity, the fragmentation scale height:

(i) $\left|H_{p} / H_{f}\right| \ll 1$ : Single-body model

where $H_{f}=$ Fragmentation scale height $\equiv-\left\{A(z) / A_{\infty}\right\} / \partial\left\{A(z) / A_{\infty}\right\} / \partial z$; $H_{p}=$ pressure scale height $\equiv-p(z) / \partial p(z) / \partial z=R T / g ; A(z)=$ frontal area as a function of height, $z ; A_{\infty}=$ initial frontal area $p(z)=$ atmospheric pressure as a function of height (ideal gas assumed); $R=R^{*} \mid M=$ gas constant for the atmosphere; $g=$ acceleration due to gravity; $R^{*}=$ universal gas constant; $M=$ mean molecular weight of the air $=28.966 \mathrm{~kg} / \mathrm{kmol}$ below $\sim 85 \mathrm{~km}$.

This limit includes a self-similar ablation solution with no shape change for $\mu \equiv 2 / 3$. The pressure scale height needs to be replaced by the density scale height in a non-isothermal, hydrostatic atmosphere. For $0 \leq \mu<2 / 3$, the solution includes ablation and deceleration, as well as shape change with the frontal cross-sectional area, $A(z)$, decreasing with decreasing height.

Stated simply the fragmentation scale height is simply the vertical distance scale over which the frontal area of the bolide increases by $1 / e$ during increasing downward penetration during entry. Since the atmospheric pressure scale height is a measure of the $e$-folding distance of the atmospheric pressure, i.e., the distance over which the air pressure decreases vertically by $1 / e$ as vertical distance above the earth increases, the relevance of a 
comparison between these two fundamental vertical lengths scales becomes more readily apparent.

(ii) $H_{p} / H_{f} \gg 1:$ Catastrophic fragmentation limit

with $\mu<0$, this solution allows for ablation and deceleration as well as shape change, but $A(z)$ increases with decreasing height. This case encompasses the so-called quasi-liquid "pancake" fragmentation model previously used by numerous authors to model the entry of Shoemaker-Levy 9 into Jupiter's atmosphere. It has already been shown in ReVelle (2001c) that the fragmentation scale height is a significant parameter (for the conditions expressed above in (5)) that can be readily be derived during an evaluation of the equations for the height of maximum luminosity during entry. Similar comments also apply to other quantities which also reach a maximum during entry such as drag and deceleration, energy deposition, the heating rate, etc.

As shown below in Table I, an additional solution is possible for $\mu>0$ where the frontal area increases with increasing penetration depth if the fragments act collectively in the wake after they are drawn forward toward the leading fragment (Sepri et al., 1981). This is a clarification from the regime list of ReVelle (2001a). For the single-body approximation with $\mu>0$, only negative fragmentation scale heights are possible. If the meteoroid has already broken with a leading fragment and $\mu>0$, it is possible to achieve a small positive fragmentation scale height, but only if there is a strong collective wake behavior among the fragments. The fragmentation scale height is always $<0$ if there is a non-collective wake behavior, i.e., if only a rapid transfer of the fragments into the wake with no further interaction with the leading fragment occurs. Intermediate behavior (oscillating periods of collective wake activity) may also produce a positive fragmentation scale height as well and this possibility is currently under investigation. The shape change parameter, $\mu$, cannot yet generally be calculated, except using detailed numerical models. The single known exception is evident in the limit in which velocity is a constant and assuming an $H_{f} / H_{p}$ ratio (which the author has derived and already presented at various conferences).

\subsection{SimPLIFIED BREAK-UP MODEL}

In our modeling we have allowed bolide break-up triggering into a progressive cascade mechanism only if the stagnation pressure exceeds the tensile/compressive or equivalently the "breaking" strength, $T_{s}$, (thermal processes are generally much too slow for the range of thermal conductivities for most observed bolide types. An exception to this may be if the fragments 


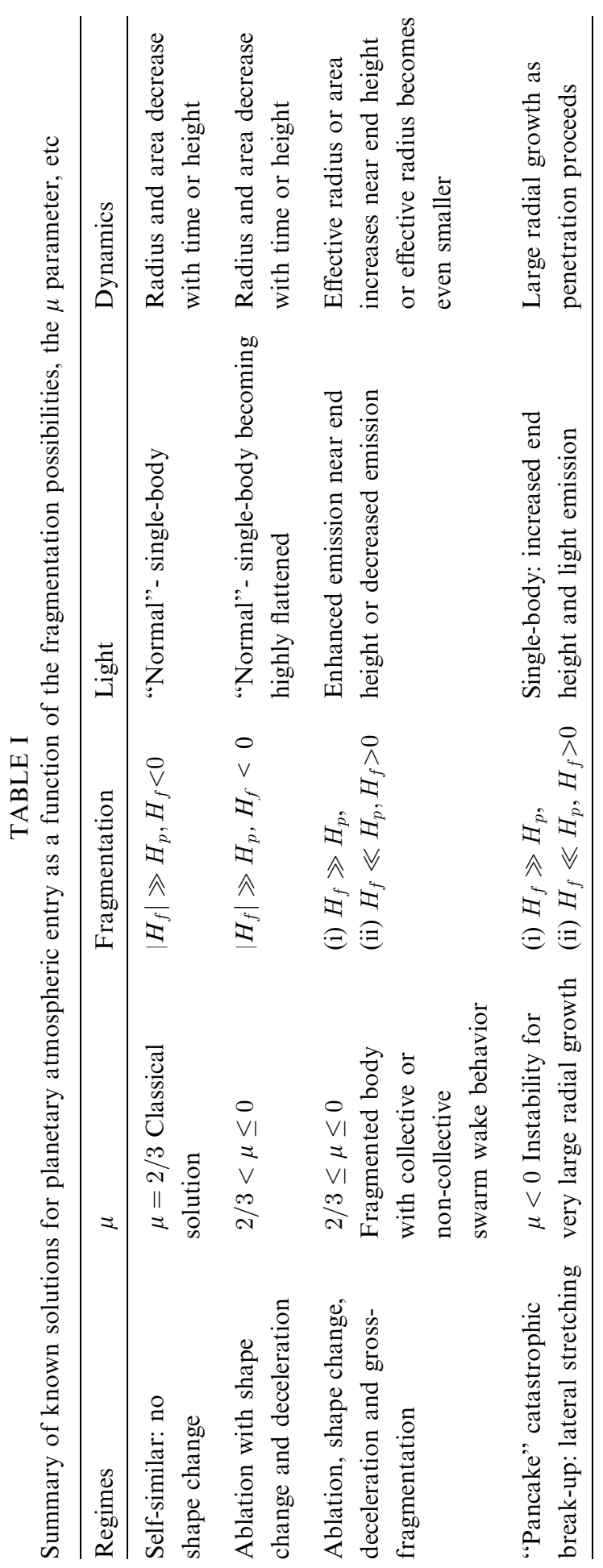


produced are small enough to allow a rapid thermal conduction into their interior during the lifetime of the luminous entry and concomitant ablation process which is generally $<\sim 10 \mathrm{~s}$ ).

Fragmentation is much like turbulence, i.e., an unsolved problem ands it is unlikely than a single universal approach will work for all bolides. We have assumed a "triggered" progressive fragmentation model and this does seem do work for a number of cases as a cascade of fragments develops much like the description of the cascade during a cosmic ray shower event. However, in a number of studied cases, this method does not seem adequate and single discrete large amplitude fragmentation events can also occur. In such cases we simply limit the number of fragments to a small number $\sim 2-4$ and continue calculating the results with the fragmentation processes subsequently turned off. Additional fragmentation models will also be attempted in the future to determine if a more general fragmentation process can be identified.

The cascade process was assumed to be a simple geometric series of the form $1 \rightarrow 2 \rightarrow 4 \rightarrow 8$, etc. pieces (other simple progressions are also easily input for this part of the fragmentation modeling process however) until some limiting value has been achieved. As will be noted later, this increasing effect is only marginally significant for deceleration, but the final number of fragments produced is extremely sensitive for the luminosity that is predicted. The final number of fragments (if break-up was triggered) was an input to the code and usually varied between 2 and 1000. For specific photographed entries we knew the final number directly from the photographs, otherwise the final number was calculated simply by trial and error estimation between model outputs and actual flight data. The breaking strength has been defined for either a homogeneous body of uniform bulk density (composition) or for a porous body, including possible effects from prior space collisions (macroscopic cracks), etc. We assigned nominal $T_{s}$ values from uni-axial laboratory test data on meteorite samples using materials from ordinary chondrites to pumice (Baldwin and Sheaffer, 1971, etc.). We assigned a reference strength for each bolide type $=k^{*} \cdot T_{s}$ with $k^{*}=0.20$ as a nominal value after accounting for weaker structure due to porosity, space collisions, etc. After break-up occurs, we allowed each fragment to be of the same size and computed $\sigma$ for the main fragment leading the swarm of fragments that are cascading into more fragments while continuing to ablate according to their size. After the body is split into two or more parts, thus decreasing the radius, $\sigma$, decreases dramatically (since it is computed for the newly broken, individual leading fragment with a much smaller radius). The gas cap boundary layer can undergo a transition from turbulent to laminar flow as time proceeds and $\sigma$ will rapidly rebuild due to increased radiative as well as convection/conduction effects and the gas cap can again become turbulent. If the progressive splitting continues and the radius decreases, $\sigma$ decreases again and the gas cap boundary layer can change from turbulent to laminar once 
again, but $\sigma$ may again rebuild and the gas cap can become turbulent again, etc.

We assumed that there was a rapid wake transfer and thus set a time delay for the fragments to reach the wake and before the start of optical radiation emission ( $\ll 1 \mathrm{~s}$ using a simple linear scheme that depends upon the instantaneous speed of the leading fragment). Once the fragments reached the wake, we allowed either a non-collective or a collective wake model to be operative (Sepri et al., 1981). In the former possibility, the fragments continue to ablate and are permanently lost from influencing the deceleration and luminosity at progressively lower altitudes. For the latter possibility the fragments are drawn forward (due to the decreased air pressure behind the main body) toward the leading fragment and act collectively as a flying "swarm" of fragments that determines the future luminosity and deceleration possibilities. It is also possible for this process to repeat on a quasi-periodic basis during entry and this intermediate fragmentation scheme is also currently being investigated. As noted in ReVelle (2002a), this relatively rapid variation of the light curve has been interpreted previously as being due to bolide rotation effects (Beech and Brown, 2000), but according to the above model, it could also be due to changes in the frontal cross-section of the body as fragments sweep into and then out of the near-wake region where significant luminosity is radiated.

Numerous authors have addressed the breakup problem, way too many to summarize in this short review. Many schemes have tried to modify the single-body model to enhance the frontal area for both drag and heat transfer or just for heat transfer, or to hypothesize a turbulent mixing process of the ablated vapor that remains with the body as it continues its subsequent flight, a "quasi-liquid" pancake model of rapid lateral spreading into a very flattened disk, porosity effects, etc. Most schemes have failed to capture the essential physics of what is actually happening during entry. This is one reason why we chose to not only predict the resulting dynamical entry deceleration and drag, but also the simultaneous panchromatic luminosity production.

In this model we have followed the pioneering work of Sepri et al. (1981) and simply allowed the fragments to assemble themselves in a maximum drag orientation fashion (an assemblage of pieces all of the same size that are by assumption flying side by side (so-called "flying buckshot") as they continue to ablate and fragment. This assumption can only be justified by additional separate numerical simulations suggested in Sepri et al. (1981). Also, as has been discussed previously in ReVelle (2001a, 2002a) this topic is currently a subject of further investigation. The fragmentation process assumed in this analysis of the progressive fragmentation type is not unique and has not found to be in agreement with the entry modeling of all bolide entries studied thus far. It does seems to be a good start at least for a large number of the 
numerous modeled bolide events for all of the modeled parameters including both the fragmentation possibilities as well as the panchromatic luminosity.

\subsection{Multiple flow Regimes}

We can define the following relevant Knudsen numbers of the flow:

(i) For atmospheric processes:

$$
K n^{* *}(z)=\lambda(z) / H_{\rho}(z)
$$

(ii) For mechanical wave generation from bolides:

$$
K n^{*}(z)=\lambda(z) / \Lambda(z)
$$

(iii) For heat and momentum transfer to bolides:

$$
K n(z)=\lambda(z) / r(z)
$$

where $\lambda(z)=$ neutral gas mean free path, $H_{\rho}(z)=$ atmospheric density scale height $\equiv\{-\rho(z) / \partial \rho(z) / \partial z\}, \rho(z)=$ atmospheric density as a function of height, $\Lambda(z)=$ wavelength of the AGW generated by the bolide, $r(z)=$ radius of the bolide.

The definition of continuum fluid properties is if the classical Knudsen number given by (6c) above is $\ll 1$, whereas the free molecule flow definition occurs for the classical Knudsen number $\gg 1$ (Bronshten, 1983). The conventional theory for heat transfer to the meteor body has consistently used definition (iii) above. Thus, we are proposing a new theory in order to include free molecular flow using (ii) above where $\Lambda=$ diffuse wavelength of the cylindrical propagating waves $\left(=k \cdot 2.81 \cdot R_{0}(z)=5.62 \cdot k \cdot M a(z) \cdot r(z)\right)$ that "must eventually pile up at infinity", unlike the strong blast waves that form uniquely for a continuum flow situation. In the above expression, $k$ is a constant at any height which accounts for non-single-body behavior of the blast wave relaxation radius, $R_{0}(z)$ and explicitly accounts for fragmentation effects $(k$ is $>1$ and usually $<5$ in the author's modeling experience which depends in part on the final number of fragments prescribed) and finally $M a$ is the body Mach number comparing the instantaneous speed of the leading body against the local adiabatic thermodynamic sound speed, $c_{s}$. Since $\Lambda(z) \propto r(z)$, this relation for $K n^{*}$ scales directly with $K n$ in a constant manner and is consistently much smaller than $K n$ due primarily to the large Mach number of the flow. Thus, we must include this term, which involves the 
differential acoustic efficiency, in the overall meteor power budget at all heights for almost all sizes of bodies because $K n^{*}<\mathrm{O}(1)$ even at the lowest penetration heights even though $K n$ is not $\mathrm{O}(1)$. Even when $K n^{*} \gg 1$, the differential acoustic efficiency is $\ll 1$ already (see below) which is probably why this fact was not recognized much earlier.

Thus, to briefly summarize, even before the classical Knudsen number is sufficiently small compared to unity, the ratio of the neutral gas mean free path to the "diffuse" blast wave wavelength is sufficiently small so that power is being deposited into relevant and propagating atmospheric waves, so that a proper accounting of the power balance demands that this form of power loss be included even for cases where the classical $K n$ is quite large.

\subsection{ENTRY PROCESSES: DRAG, DECELERATION, LUMINOSITY PRODUCTION AND ENERGETICS, ETC.}

We have started from a fundamental ad hoc assumption, namely that the bolide produced luminosity is proportional to the time rate of change of the kinetic energy of the body (single-body or fragmented), with the proportionality constant being the luminous efficiency factor, $\tau_{L}$, in the spectral band of interest, i.e., the panchromatic band from $~ 360-675 \mathrm{~nm}$ (Spurny et al., 2001).:

$$
I(t)=-\tau_{L} \cdot d E(t) / d t
$$

This assumption is justified on the basis of bolide spectra which show primarily that excited lines due to excited meteor atoms/ions are present with generally only a small contribution from excited atmospheric species at least in a panchromatic pass-band. The luminous efficiency is now available from a refined semi-empirical model (ReVelle and Ceplecha, 2001f, 2002b). We present below an example of our recent successful luminosity modeling effort for the famous Czech bolide, Benesov (May 7, 1991) in Figure 4. This bolide was considered previously by Borovicka and Spurny (1996), Borovicka et al. (1998a, b), etc.

Our present results confirm the previous initial mass estimates for Benesov of $\sim 2000 \mathrm{~kg}$ while providing far more reasonable estimates of its terminal mass than predicted in previous theoretical modeling studies which were all greater then at least $500 \mathrm{~kg}$. At the same time we have also provided a very reasonable zeroth order fitting of some of the key details of its observed light curve.

\subsubsection{Homogeneous versus porous meteoroid modeling}

ReVelle (2001e, 2002b) has shown that at the same velocity, mass, entry angle, shape, shape change parameter, etc., porous bolides are far more 
efficient light producers than are low porosity bodies. Specifically, light increases compared to non-porous bodies are for Group II (50\% porosity) - up to 9 times larger, for Group IIIA (75\% porosity) - up to 51 times larger, and for Group IIIB (91\% porosity) - up to 225 times larger. The increase in light production is due physically to the fact that the ratio of the kinetic energy changes of a porous body compared to that of a non-porous body increase as the ratio of the square of the heat transfer area compared to the drag area. Consequently, as shown in ReVelle (2001e) the light production increases as the ratio of the square of the ablation coefficient for porous bodies compared to those for non-porous bodies. These values have assumed that all meteor bodies are chondritic in their composition. It has already been demonstrated that for bodies such as the Tagish Lake bolide and concomitant meteorites (Brown et al., 2002), porosity modeling is totally essential in order to correctly match the dynamics, the energetics as well as its luminosity.

Other effects such as bolide rotational influences on the light production are discussed in ReVelle (2002a).

\subsection{TOTAL POWER BALANCE: DIFFERENTIAL AND INTEGRAL EFFICIENCIES}

Bolide source energies can be estimated in a number of ways. These include various entry modeling (ReVelle, 1979, 1980, 2001a, 2002a) and by using infrasonic/seismic techniques, etc. Most of the infrasonic approaches are listed in ReVelle and Whitaker, (1999). There is also a very promising semiempirical approach to the amplitude prediction problem combining spaceborne sensor detections, used in combination with infrasonic detections, in Edwards et al. (2004 - this issue). Still additional applications of the acoustic differential efficiency approach combined with acoustic-gravity wave conservation (see below) have lead to a new method for very reliably predicting the source properties from satellite or ground-based camera data as well as infrasound and/or seismic detections as well at moderately close ranges (ReVelle et al., 2004).

\subsubsection{Differential and integral efficiencies}

2.6.1.1. Large bolides: total power budget analyses: We can perform a power budget analysis at any instant of time or equivalently evaluate the time rate of change of the kinetic energy of the bolide $\left(\equiv 1 / 2 \cdot V^{2} \cdot d m / d t+m V \cdot d V / d t\right)$, as originally presented in equation (1) of Romig (1965), but that definitely includes the differential acoustic efficiency, $\varepsilon$, (that is discussed further directly below) as long as $K n^{*}<\mathrm{O}(1)$ as justified earlier in Section 2.4. Multiple Flow Regimes:

$$
P_{\text {tot }}=\sum P_{\mathrm{i}}=P_{\text {heat }}+P_{\text {light }}+P_{\text {sound }}+P_{\text {ionization }}+P_{\text {dissociation }}+\cdots
$$


or:

Total power dissipated $=$ thermal power $($ heat dissipated $)+$ light power + acoustic power + ionization power + dissociation power $+\cdots$

Algebraic normalization, at any instant, of this relative partitioning of various complex energetics processes produces:

$$
1=P_{\text {heat }} / P_{\text {tot }}+P_{\text {light }} / P_{\text {tot }}+P_{\text {sound }} / P_{\text {tot }}+P_{\text {ion }} / P_{\text {tot }}+P_{\text {diss }} / P_{\text {tot }}+\cdots
$$

We can identify each of these ratios as the efficiency of the power dissipation for each of the recognized physical processes during entry as:

(1a) Luminous efficiency $=P_{\text {light }} / P_{\text {tot }}$ (over optical wavelengths); or: (1b) radiative efficiency $=P_{\text {radiation }} / P_{\text {tot }}$ (over all wavelengths); (2) Acoustic efficiency $=P_{\text {sound }} / P_{\text {tot }}$; (3) ionization efficiency $=P_{\text {ion }} / P_{\text {tot }}$; (4) dissociation efficiency $=P_{\text {diss }} / P_{\text {tot }}$; Here we have not differentiated between the dissociation of the air molecules and that of the meteoric vapor itself in this zeroth order energetics evaluation process.

These are differential efficiencies at any point along the entry path.

This evaluation process was initially described by Opik (1958) and Romig (1965) with respect to the reference frame of the moving bolide for free molecular flow in the form: "the relative kinetic energy of the intercepted air particles is transferred to the body in the form of heat and the materials ejected from the surface produced light and ion pairs upon collision with ambient air particles". Obviously the complexity of continuum flow and additional aerodynamic regimes are far more complicated to evaluate reliably, but we seem to be doing a reasonable job of evaluating all of the known processes, with the exception of a few "small" omissions discussed directly below.

In this preliminary power budget result not all physical processes have yet been included, i.e., some of the neglected processes include: Atmospheric internal gravity wave excitation, infrared and microwave luminosity production, surviving meteorite fragment kinetic energies, etc. For this reason the sum of the algebraic expression in (9) above should sum to unity only approximately. This procedure was developed primarily as a theoretical zeroth order guide purely to examine if the principle energetics components modeled during entry were being satisfactorily accounted for such that a gross omission of an energetics source (or sink) term was being overlooked.

2.6.1.2. Differential acoustic efficiency definition: The near-field differential acoustic efficiency, $\varepsilon$, can be evaluated by forming the ratio of the weak shock, acoustic wave kinetic energy density (at $x=10$ ) compared to the bolide kinetic energy density deposition into the non-linear volume defined at 
$x=1$, where $x \equiv R / R_{0}, R=$ slant range from the bolide and $\mathrm{R}_{0}$ is the line source/modified line source blast wave relaxation radius all as a function of the geopotential height, $z$ (approximately equal to the geometric height within $\sim 1 \mathrm{~km}$, below about $80 \mathrm{~km})$.

Let:

$\varepsilon \equiv$ wave kinetic energy density/kinetic energy transferred into the nonlinear deposition volume

$$
\varepsilon(z) \equiv 1 / 2 \cdot \rho(z) \cdot \Delta u^{2}(z) /\left\{1 / 2 \cdot m(z) \cdot V^{2}(z) /\left\{\pi \cdot R_{\mathrm{o}}^{2}(z) \cdot l(z)\right\}\right.
$$

$\Delta u=\Delta p(z) /\left\{\rho(z) \cdot c_{s}(z)\right\}$ for plane acoustic waves - wind due to the wave $p(z)=$ ambient pressure as a function of altitude, $\rho(\mathrm{z})=$ ambient air density as a function of altitude, $l(z)=$ line source length as a function of altitude, $l(z) \equiv\left(z^{\prime}-z\right) / \sin \theta ; \varepsilon$ is evaluated at $x=10\left(=10 \cdot R_{0}\right.$ from the entry trajectory) where $\Delta p(z)=0.0575 \cdot p(z)$ from "first principles", theoretical numerical line source pressure wave calculations as discussed in ReVelle $(1976,2002 a)$ and

see also further details below.

We can also perform a units analysis for $\varepsilon$ to show that as defined it is dimensionless and write this expression out in the scaled form:

$$
[\varepsilon]=\left(1 / \gamma^{2}\right) \cdot\left\{\left(\rho(z) / \rho_{m}\right) \cdot\left(V(z) / c_{s}(z)\right) \cdot\left(l(z) /\left(R_{0}(z)\right)\right\}\right.
$$

Specific final numerical values are as follows:

$$
\varepsilon=k^{\prime} \cdot p^{2}(z) \cdot l(z) \cdot V(z) /\left(\rho(z) \cdot \rho_{m} \cdot c_{s}^{5}(z) \cdot R_{0}(z)\right)
$$

where $k^{\prime}=0.0198$ as evaluated for $x=10$ (or $k^{\prime}=0.0163$ for $x=5$ ).

The evaluation of $k^{\prime}$ is to be accomplished where the waves are quasilinear so that non-linear effects are sufficiently small (very small amplitude, etc.) as indicated in ReVelle (1976). The first position for which this is the case, is at $x=10$ (with a slight $x$ dependence noted for the value of $k^{\prime}$ ).

To evaluate the general case of acoustic-gravity wave efficiency including IGW (see also Section 3 below) we have derived results from additional information in Mihalas and Weibel-Mihalas, 1999) with $\gamma=$ the ratio of the specific heat at constant pressure to that at constant volume for air (considered as an ideal diatomic gas $\cong 1.40$ ):

$$
\Delta u=\left[c_{s}^{2} \cdot K_{x} /(\gamma \cdot \omega)\right] \cdot\left[\left\{\Delta p / p_{0}\right\}\right] ; \quad \omega=2 \cdot \pi \cdot f ; \quad K_{x}=2 \cdot \pi / \lambda_{x}
$$

where $K_{x}=$ horizontal wavenumber of the wave, $\omega=$ angular wave frequency $=2 \pi \cdot f, f=$ linear wave frequency, $\lambda_{\mathrm{x}}=$ horizontal wavelength.

This is clearly a topic for further research in order to compare results against the work of Golitsyn et al. (1977) - see below in Section III for further details. 
2.6.1.3. Definition of the integral acoustic efficiency: $\varepsilon_{\text {int }}=$ acoustic kinetic energy density/(initial kinetic energy/total volume of energy deposition)

$$
\begin{aligned}
& \varepsilon_{\text {int }} \equiv 1 / 2 \cdot \rho(z) \cdot \Delta u^{2}(z) /\left\{1 / 2 \cdot m_{\infty} \cdot V_{\infty}^{2} /\left\{\pi \cdot R_{0 \infty}^{2} \cdot l_{\infty}\right\}\right. \\
& \varepsilon_{\text {int }} \equiv k^{\prime} \cdot\left\{p^{2} /\left(\rho(z) \cdot c_{s}^{5}(z)\right\} \cdot\left\{l_{\infty} / R_{0 \infty}\right\} \cdot\left\{V_{\infty} / \rho_{m}\right\}\right.
\end{aligned}
$$

where once again $k^{\prime}=0.0198$ as evaluated for $x=10$.

2.6.1.4. Differential and integral acoustic efficiency: their relationship

$$
\begin{gathered}
\varepsilon / \varepsilon_{\text {int }}=1 / 2 \cdot \rho(z) \cdot \Delta u^{2}(z) /\left\{1 / 2 \cdot m(z) \cdot V^{2}(z) /\left\{\pi \cdot R_{0}^{2}(z) \cdot l(z)\right\}\right. \\
/\left[1 / 2 \cdot \rho(z) \cdot \Delta u^{2}(z) /\left\{1 / 2 \cdot m_{\infty} \cdot V_{\infty}^{2} /\left\{\pi \cdot R_{0 \infty}^{2} \cdot l_{\infty}\right\}\right]\right. \\
\varepsilon / \varepsilon_{\text {int }}= \\
k^{\prime} \cdot p^{2}(z) \cdot l(z) \cdot V(z) /\left(\rho(z) \cdot \rho_{m} \cdot c_{s}^{5}(z) \cdot R_{0}(z)\right) \\
/\left[k^{\prime} \cdot\left\{p^{2}(z) /\left(\rho(z) \cdot c_{s}^{5}(z)\right\} \cdot\left\{l_{\infty} / R_{0 \infty}\right\} \cdot\left\{V_{\infty} / \rho_{m}\right\}\right]\right. \\
\varepsilon\left(z_{\text {end }}\right) / \varepsilon_{\text {int }}=\left\{l\left(z_{\text {end }}\right) / l_{\infty}\right\} \cdot\left\{V\left(z_{\text {end }}\right) / V_{\infty}\right\} \cdot\left(R_{0}\left(z_{\text {end }}\right) / R_{0 \infty}\right\}
\end{gathered}
$$

So far in our panchromatic pass-band power budget analyses we have not specifically included either the infrared or the ultraviolet radiative emission from bolides or the microwave electrophonic/ethaerial sound emission or the internal gravity wave differential efficiency, or the efficiency corresponding to the kinetic energy of the surviving fragments, etc. The ultraviolet emission is very important however, but has been implicitly included already in our analysis since the ablation calculations (through $\sigma(z)$ ) have specifically included the radiative heat transfer from the very strong leading shock front. Since our "total" power balance (see below) is so close to $100 \%$ at low heights, it is unlikely that the other differential efficiencies are generally very large. The electrophonic/ethaerial microwave source is known to be very small for example.

2.6.1.5. Differential/integral luminous efficiency definition: This differential luminous efficiency is defined as the optical luminous power (Watts/steradian) as a function of range compared to the time rate of change of the bolide kinetic energy in a panchromatic pass-band: $\sim 360-675 \mathrm{~nm}$ (with the integral luminous efficiency production being compared to the initial kinetic energy).

The differential luminous efficiency expression developed by ReVelle and Ceplecha (2001f) is given in two separate velocity regions and is plotted in 
two velocity difference regimes in Figure 2 (near the beginning of the flight) and in Figure 3 (closer to the end of the luminous flight).

We now will determine the generalized relationship between the differential and integral luminous efficiencies (work that originated in ReVelle (1981)):

2.6.1.6. Differential luminous efficiency values:

$$
\begin{aligned}
& I_{L}(t)=-\tau_{L} \cdot d E_{k} / d t \\
& \tau_{L}=-I_{L}(t) / d E_{k} / d t
\end{aligned}
$$

2.6.1.7. Integral luminous efficiency values:

$$
\int I_{L}(t) d t=-<\tau_{L}>\cdot d E_{k}(t)
$$

\section{Semi-empirical luminous efficiency (\%): Panchromatic pass-band; ReVelle and Ceplecha (2002): $0 \%$ porosity}

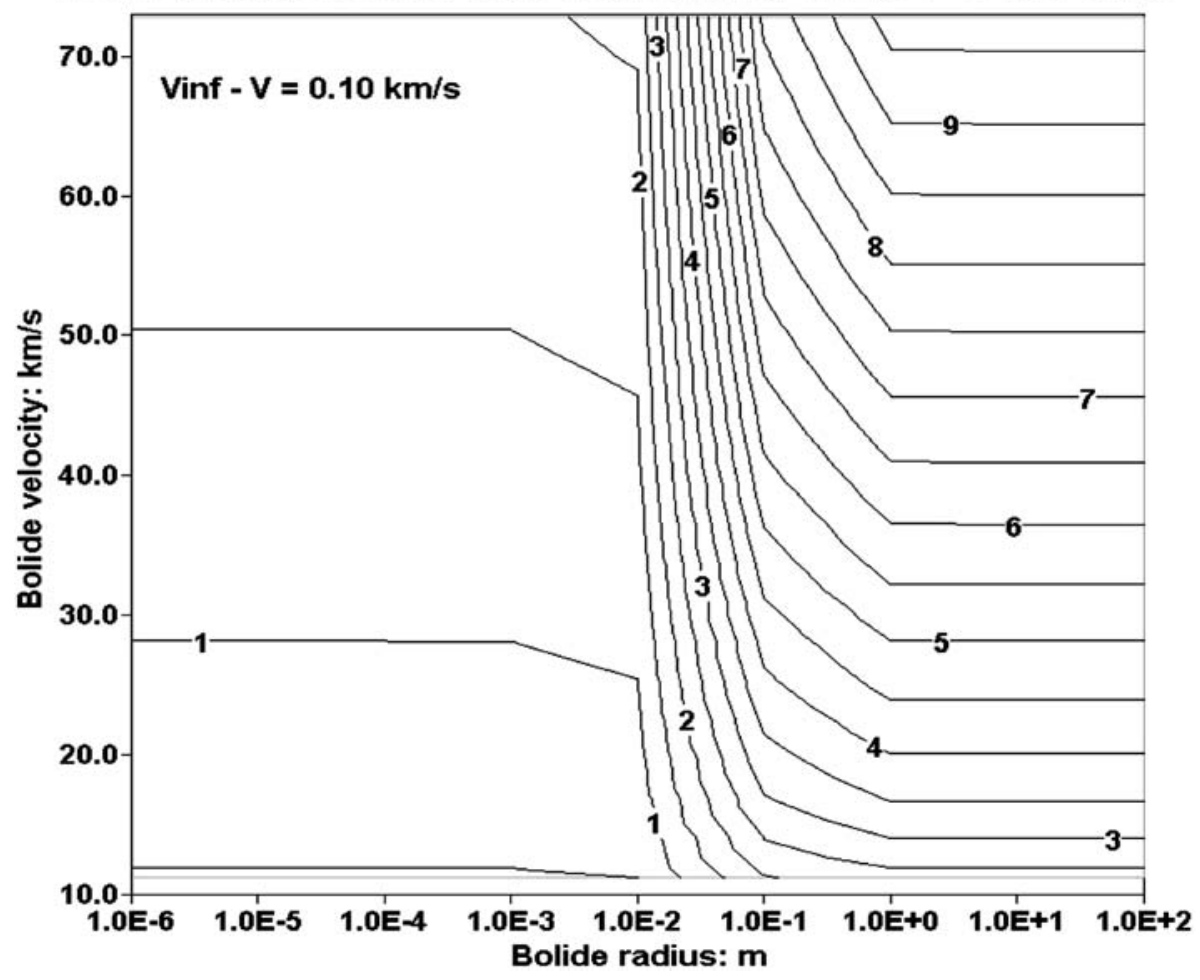

Figure 2. Panchromatic luminous efficiency as a function of mass, air density and velocity near the beginning of the atmospheric trajectory. 


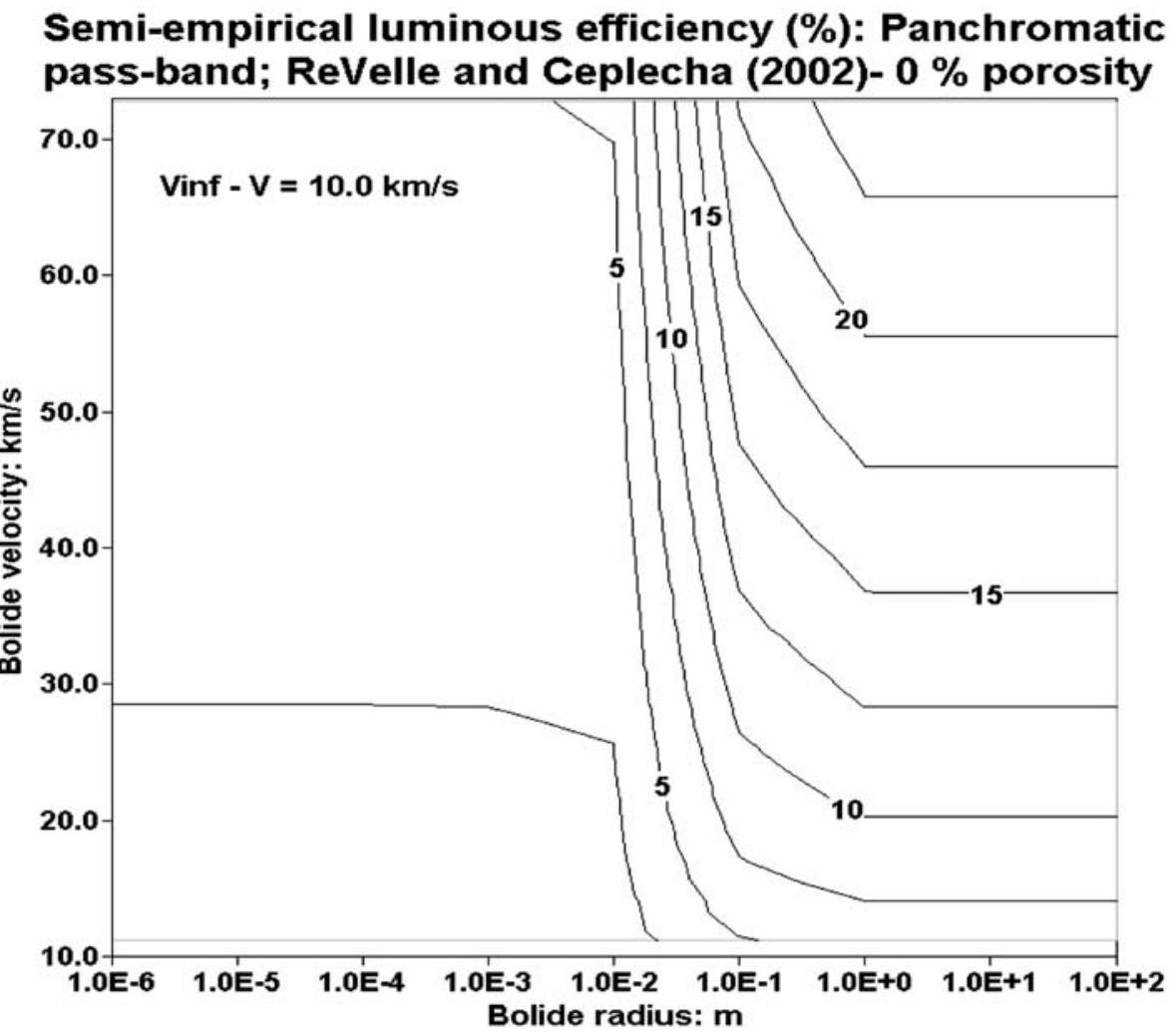

Figure 3. Panchromatic luminous efficiency as a function of mass, air density and velocity near the end of the atmospheric trajectory.

$$
\begin{aligned}
& \int I_{L}(t) d t=-<\tau_{L}>\cdot\left\{0.5 \cdot\left(m_{\infty} \cdot V_{\infty}^{2}-m_{f} \cdot V_{f}^{2}\right)\right\} \\
& <\tau_{L}>=-\int I_{L}(t) d t /\left\{0.5 \cdot\left(m_{\infty} \cdot V_{\infty}^{2}-m_{\mathrm{f}} \cdot V_{f}^{2}\right)\right\}
\end{aligned}
$$

2.6.1.8. Generalized final results:

$$
\tau_{L} /<\tau_{L}>=\left\{I_{L}(t) /\left[2 \cdot \int I_{L}(t) d t\right]\right\} \cdot\left\{\left(m_{\infty} \cdot V_{\infty}^{2}-m_{f} \cdot V_{f}^{2}\right) / d E_{k} / d t\right\}
$$

and where the integration limits in (17a)-(17c) are over the visible duration of the bolide, i.e., $t=0$ at $z=z^{\prime}$ to $t=t_{\text {end }}$ at $z=z_{\text {end }}$ where $z_{\text {end }}$ is the lowest height where luminosity has been registered by the photographic emulsion 


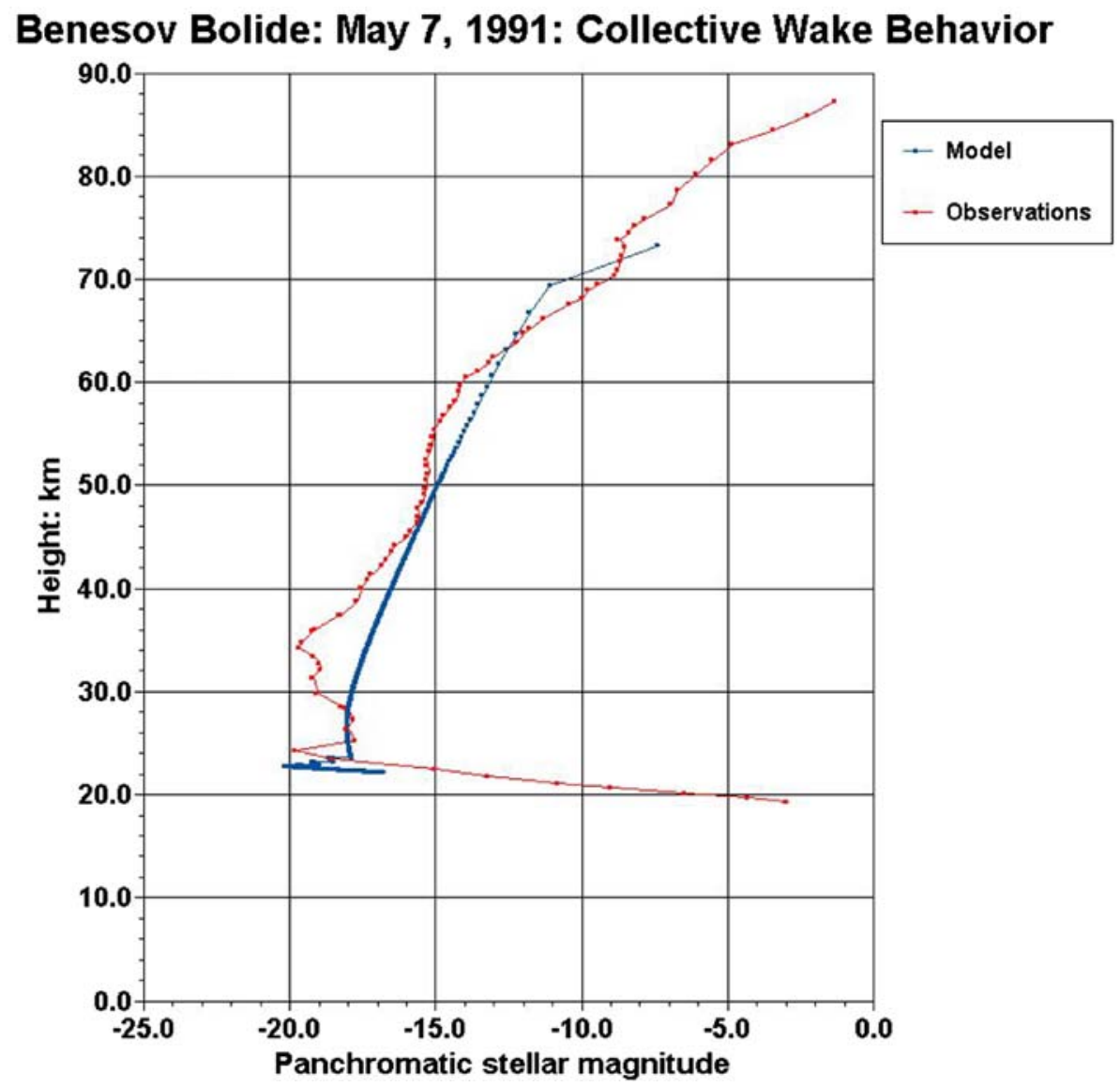

Figure 4. Example of panchromatic luminosity modeling prediction for Benesov (entry parameters assigned: sphere of unchanging shape, initial radius $=0.50 \mathrm{~m}$, Zenith angle of the radiant $=9.4^{\circ}$, initial velocity $=21.8 \mathrm{~km} / \mathrm{s}$, uniform volume porosity $=15 \%$, Final number of fragments $=8$, collective wake model behavior assumed for an initial mass of $=1647 \mathrm{~kg}$ and a predicted terminal mass $=61.02 \mathrm{~kg}$ ).

(for the case $D=4.605$, this is also the corresponding height where $99 \%$ of the original kinetic energy of the bolide has been removed).

Thus, we can see that there is not a general constant relationship between these two parameters, since each case will depend upon the specific values of mass, degree of porosity, ablation parameter, luminous efficiency, etc.

\subsubsection{Total power budget analysis}

2.6.2.1. Evaluation of dimensionless efficiency ratios: To perform a total power budget for the bolide entry process, we have started our analysis from two detailed and independent sets of results, namely the differential acoustic efficiency discussed above and the semi-empirical, panchromatic differential 
luminous efficiency: (ReVelle and Ceplecha, 2001f, 2002c). In order to accomplish the total power budget, we have scaled all of the differential efficiencies, except for the differential acoustical efficiency, with respect to the semi-empirical, panchromatic luminous efficiency utilizing data from earlier theoretical and experimental measurements reported in Romig (1965) and utilized in the bolide energetics formulation presented in ReVelle (1980, 1997). Most of these energy scaling estimates were adopted from the synthesis provided by Greenhow and Hawkins (1952), most of which was based on radar and optical luminosity measurements as well as theoretical work at that time by a number of workers, including Opik. The original results of Greenhow and Hawkins were further separated into values of the various differential efficiencies for the extreme limits of "bright" and "faint" meteors. Here we have only utilized the "bright" meteor ratios and modified them in such a way as to optimize the total power budget as best as possible using the simplest possible formulation during the entire entry period.

After many sets of evaluations of these ratios by trial and error, the final, zeroth order and most simplified, normalized set of values utilized in this paper are given by

$$
\tau_{h} / \tau_{L}=20.0(*) ; \tau_{i} / \tau_{h}=0.001 ; \tau_{\text {diss }} / \tau_{h}=0.50 \quad \quad(18 \mathrm{a}, 18 \mathrm{~b}, 18 \mathrm{c})
$$

where $\tau_{h}=$ differential heat (thermal) efficiency, $\tau_{i}=$ differential ionization efficiency, $\tau_{\text {diss }}=$ differential dissociation efficiency (of the entire mixture, i.e., air and meteoric vapor).

(*) Literature values range from $\sim 50$ to $>100$ times depending on the brightness of the meteors and the spectral pass-band of the measurements, but in those evaluations, dissociation effects were not explicitly considered.

The ratios used in this paper are of course only an approximation to the "truth" and as acknowledged in the original reference of Greenhow and Hawkins (1952), the individual ratios could be uncertain by $\pm 50 \%$. We have provided this evaluation for our entry modeling, so that for a given bolide, an estimate can be made of how well we have accounted for all forms of energy at all heights/times. In this process, we are only using a semi-empirical estimate of the differential panchromatic luminous efficiency and all ratios are scaled to this efficiency with the exception of the differential acoustical efficiency. Although the semi-empirical panchromatic luminous efficiency is very precise and has been constructed to be a function of mass, air density and velocity for all possible altitudes, masses and speeds, it is not perfect in its evaluation of the bolide light emission process which is certainly very complex, especially during fragmentation events. For very low velocity entries for example, the summation of efficiencies above is typically slightly less than unity throughout the entry. 
The differential acoustic efficiency was also independently derived from "first principles, i.e., a detailed numerical solution of the fundamental conservation equations for a line source explosion" and was evaluated using the results of Plooster $(1968,1971)$ for a very high temperature and heavily ionized lightning channel. His modeling work was subsequently applied to pressure wave signals emanating from a low altitude lightning discharge by Jones et al. (1968), i.e., ordinary thunder. However, the differential acoustic efficiency is also subject to the uncertainty of the actual initial conditions present during the generation of line source blast waves at large altitudes typical of bright bolide entry as well. As noted in ReVelle (1976), the decay of the pressure amplitude with range has been written only for the case " $\mathrm{C}$ " $=1$ and " $\delta "=1$ (where " $C$ " = Plooster's adjustable parameter which determines the spatial region in which the transition to a weak shock wave occurs and " $\delta "=$ efficiency with which line source blast waves are generated in comparison to earlier results by Lin (1954)), etc.

In summing these ratios in a simple power balance, there is also the question of the spectral limits of the evaluation. Here we have limited the results to a panchromatic band $(\sim 360-675 \mathrm{~nm})$, but have appropriately incorporated information from processes occurring outside that spectral band, i.e., the ultraviolet regime for the leading very high temperature shock front and its subsequent effect on meteor ablation processes. For these and additional reasons the summation is only approximate and can at times either exceed or even be substantially less than unity, as evaluated only to zeroth order. For very large sources (very large blast wave radii) the former is almost never the case however, even for the zeroth order ratios and even at quite early times in the entry. The above ratios do appear to be consistently deficient at very early times in the free-molecule flow regime, even for very large bodies however, where the summation can often be as low as only 0.40 . We are continuing to evaluate these normalized ratios for the best possible power budget solutions for all sizes, speeds, and corresponding kinetic energies of observed bolides and will report the details of these more precise evaluations at a later time.

An example of the results of computing the total power balance versus time for the entry of the Neuschwanstein meteorite fall (ReVelle et al., 2004) is given below in Figure 5.

\subsection{SOLUTION PROCEDURE}

In order to solve the above system of equations in a self-consistent manner, we first specified all of the initial values, constants and height variable quantities such as the bolide entry speed, size, shape factor, drag coefficient, $D$ parameter, $\sigma, \theta, S_{f}, \mu$, etc. 


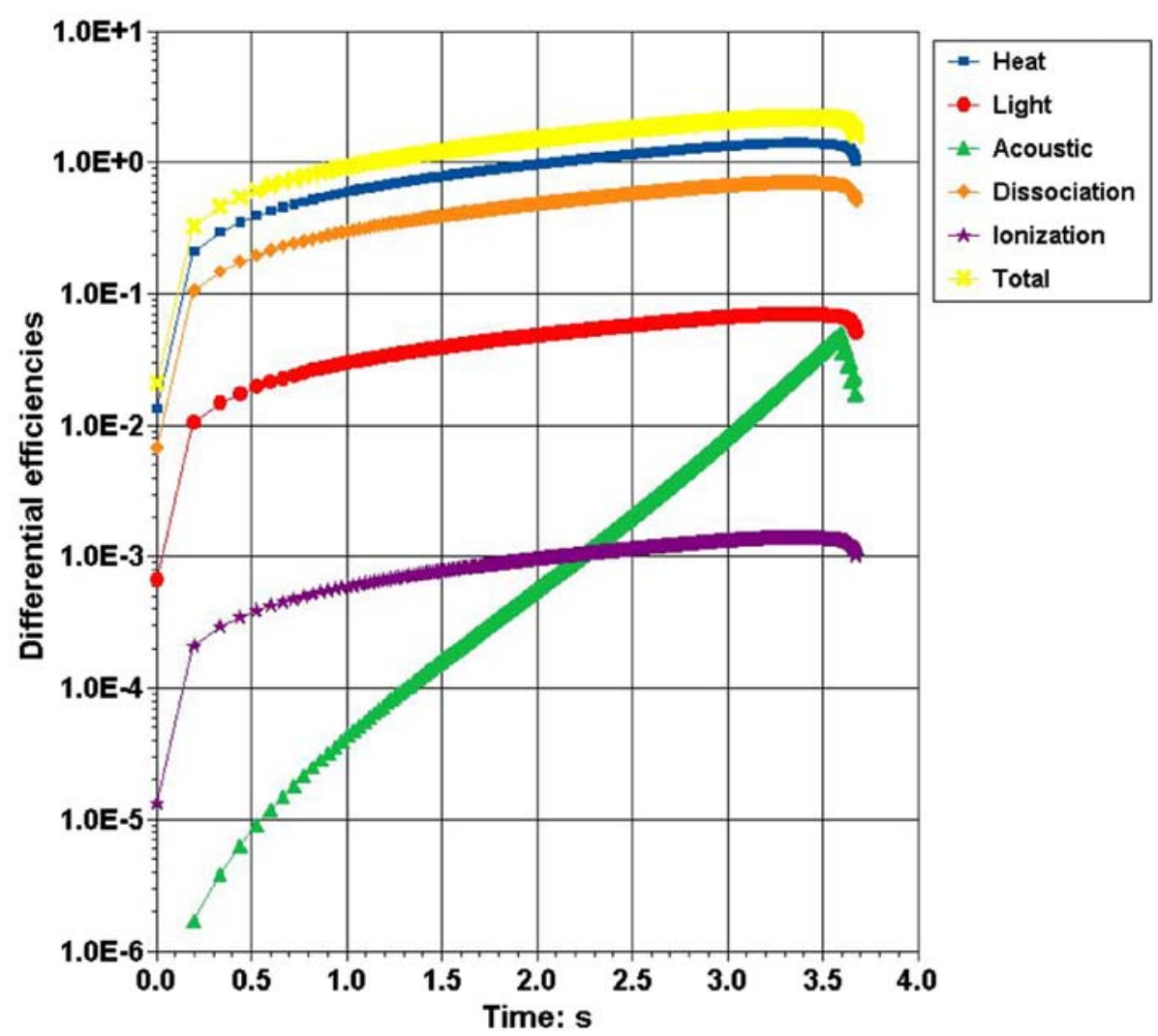

Figure 5. Total power balance in a panchromatic passband versus time for the Neuschwanstein meteorite fall (ReVelle et al., 2004).

Next we solved the transcendental equation of ReVelle (1993) and also presented in Appendix A below for speed at the height corresponding to

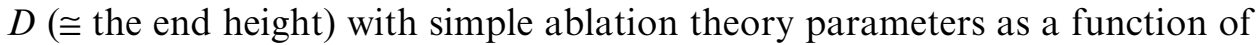
the fireball group ( $\sigma=$ constant approximation only). This is given directly below in the form:

$$
V(z)=V_{\infty} \cdot \exp \left[(\sigma / 4) \cdot\left(V_{\infty}^{2}-V^{2}(z)\right)\right] \cdot \exp [-D / 2]
$$

This simple equation is perhaps one of the weakest links in the modeling process, but fortunately the final results are not extremely sensitive to this result. It is certainly superior to arbitrarily assigning a velocity below which ablation ceases to the modeling process as has been done by some other authors however (Baldwin and Sheaffer, 1971, etc.).

Next we computed the various diagnostic heights where the maximum energy, etc. transfer occurs (ReVelle, 2002a) to help guide the modeling results and to check various regimes of validity since the entire modeling 
process is a numerical modeling effort and subject to various well known numerical instabilities, etc.

As noted in ReVelle (1979), we computed the small downward height step interval, $\Delta z$ as a function of the instantaneous meteor velocity over sufficiently small velocity increments to preserve the constant ablation parameter solution (ReVelle, 1979). We continued with a single-body type approach until fragmentation was triggered if the stagnation pressure exceeded the specified breaking strength of the body.

We utilized sufficiently small velocity steps so that $\{(1 / \sigma(z)) \cdot \partial$ $\sigma(z) / \partial z \cdot \delta z\} \ll 1$ was maintained throughout the entry. During this evaluation process, there is a specific dependence that is related to the flow regime type (body size dependence and bulk density dependence), flight velocity, entry angle, shape, meteoroid composition, mean volume porosity, etc. and these various dependencies extend throughout all parts of the modeling simulations.

If breakup was predicted to occur, we allowed progressive fragmentation of the body after a specified time lag for transfer of fragments to the nearwake, so that splitting into an increasing number of equal size pieces could occur. This cascade is continued either the body impacts the ground (or until the final number of fragments specified has been exceeded). After the rapid wake transfer, we allowed the fragments to either always remain in the wake while continuing to ablate (non-collective wake limit) or we allowed the fragments to rapidly migrate forward in the low density air to join the main mass as a collective wake that is composed of a porous, large group of bodies also undergoing ablation as in Sepri, et al. (1981). Finally, we computed the fragmentation scale height as a function of height and of $\mu$ for the conditions at all heights below the breakup height. This allowed us to evaluate whether single-body model or a fragmentation type approach was most needed.

The overall model results, given the complexity of the unknowns of both the atmosphere and of the bolides themselves, are of sufficient quality that they should be considered as just as reasonable or in some cases even better than (in terms of all the modeled variables) other previously developed theoretical models.

\section{Mechanical Wave Generation and Wave Propagation}

During the penetration of a sufficiently large body into a planetary atmosphere, mechanical waves are continuously generated. This process can be captured by using the Knudsen number that was defined earlier. This new parameter is plotted in Figure 6 along with the classical $K n$ value. Entry conditions for this vertical entry case included an initial radius $=10^{-4} \mathrm{~m}$ (sphere of unchanging shape), entry velocity $=22 \mathrm{~km} / \mathrm{s}, 50 \%$ porosity (with respect to a density of $3.70 \cdot 10^{3} \mathrm{~kg} / \mathrm{m}^{3}$ ), $D=4.605$, etc. 


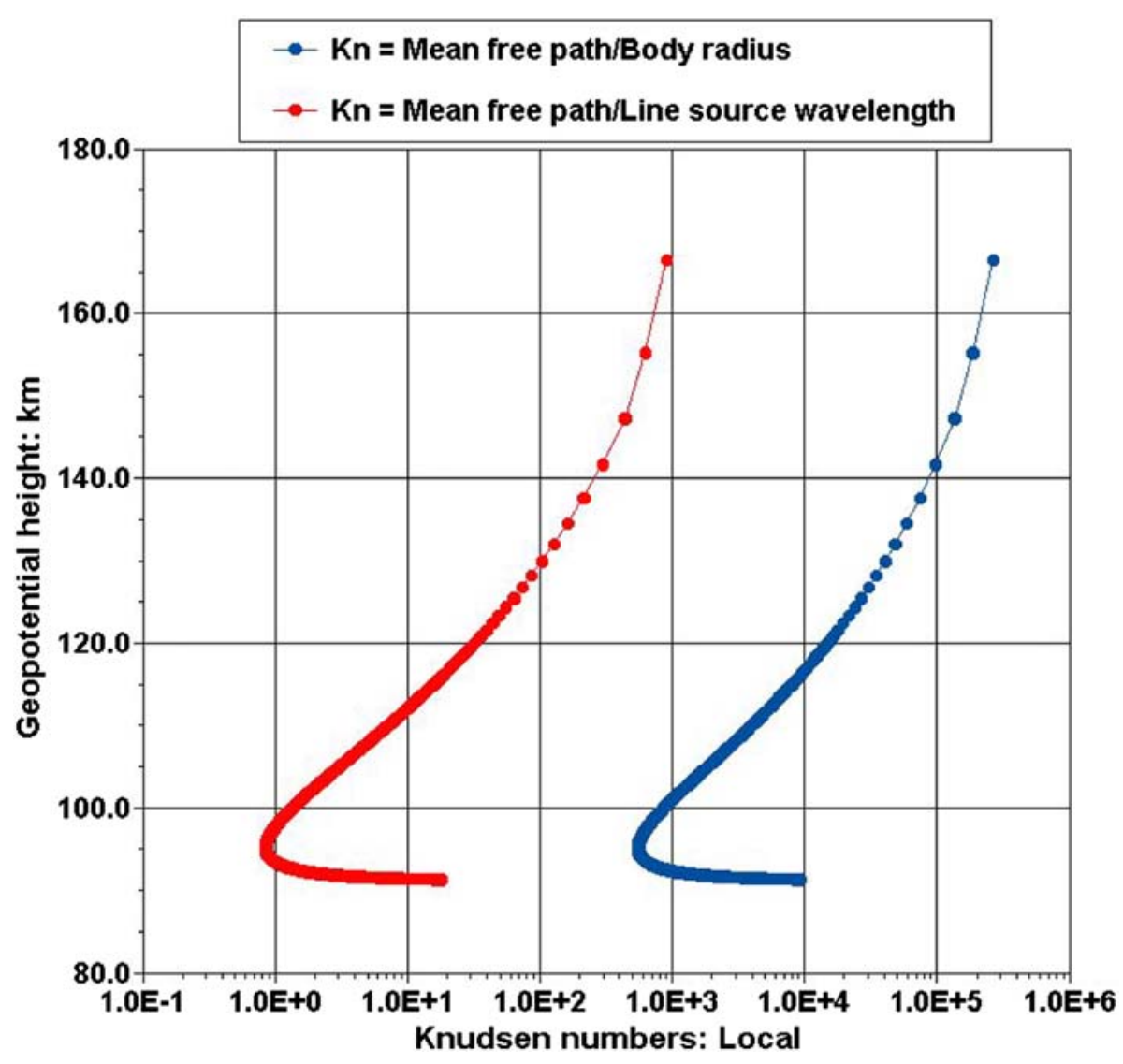

Figure 6. The modified Knudsen number and the classical Knudsen number as a function of height for very small entering meteoroids (with the classical Knudsen number $\gg$ modified Knudsen number - for details see the text).

This parameter was invented to evaluate the total power balance for small meteoroids (for which $K n$ is not in continuum flow, but for which $K n^{*}$ is definitely in the continuum flow regime). Utilizing $K n^{*}$ and the $\varepsilon$, a total power balance can be nearly achieved, even for very small meteoroids. These very diffuse, line source, high altitude blast waves are spread out over a much broader region than their counterpart at lower continuum flow heights and are a necessity if the power balance is to be satisfied at all heights for smaller meteoroids.

\subsection{Meteoroid wave source models: "Airwave” objects}

We will analyze the infrasound and to a lesser extent, the IGW from bolides using the following conceptual blast wave source models: 
(a) Idealized line source model for an infinite velocity bolide (in the no deceleration limit): The Mach cone half angle $\equiv 0^{\circ}$ so that only a highly direction cylindrical radiation pattern of AGW's is envisioned. This pattern is so directional that bolides entering steeply will subsequently have much of their wave energy refracted upward.

(b) Modified line source (due to fragmentation effects): There can be significant local ripples in the wavefront from fragmentation effects along the entry path. In the extreme gross-fragmentation limit, a protruding "omni-directional head" (for a rapidly moving point source with acoustic radiation generated in the form of quasi-spherical waves) will also lead the rearward regime of an extremely narrow type (a) line source Mach cone.

(c) Supersonic source: Non-zero Mach cone half angle whose value depends on the local sound speed and upon the instantaneous velocity of the entering meteoroid. In this case significant deceleration has occurred and very complicated acoustic radiation and subsequent refraction patterns can result (see below).

Data generated by these bodies during hypersonic entry into the earth's atmosphere were not anticipated by monitoring networks and came to be known as "airwave" objects (ReVelle, 1997).

Finally, as discussed in ReVelle (1976, 2001d), using model (a) above, there is a minimum infrasonic detection threshold for bolides corresponding to a blast radius $>\sim 10 \mathrm{~m}$ (Kraemer and Bartman, 1981). This corresponds to a minimum bolide panchromatic luminosity from $\sim-5$ to -6 or brighter in order to be detectable at ground level by an array of conventional pressure wave sensors. Expressed in terms of the bolide kinetic energy, this corresponds to values between $10^{-6}$ and $10^{-5} \mathrm{kt}$ (or 2-20 pounds of TNT equivalent energy release).

\subsection{Atmospheric AGW modeling and enERgEtic propagation inVARiants}

From the work of Golitsyn et al. (1977), in general there are four relevant atmospheric resonant frequencies even in the simplest possible atmospheric model, i.e., for an isothermal, hydrostatic atmosphere (only three of which are independent for a constant value of $\gamma$ ):

$$
\begin{aligned}
& \omega_{\mathrm{co}}^{2}=\left\{c_{s} /\left(2 H_{p}\right)\right\}^{2} \\
& \omega_{\mathrm{co}}^{2}=\left\{\gamma / 2 /\left(g / c_{s}\right)\right\}^{2}=\text { acoustic waveguide cut-off frequency squared }
\end{aligned}
$$




$$
\begin{aligned}
\omega_{a}^{2}= & g /\left(2 H_{p}\right)=\{\gamma / 2\} \cdot\left\{g / c_{s}\right\}^{2}=\omega_{\mathrm{co}}^{2} /(\gamma / 2) \\
\omega_{g}^{2}= & (\gamma-1) \cdot\left(g / c_{s}\right)^{2}=\text { Isothermal Brunt-Vaisalla frequency squared } \\
& \quad(\text { or the square of the internal gravity wave cut-off frequency) } \\
\omega_{c}^{2}= & \omega_{g}^{2} \cdot \cos ^{2} \theta^{\prime}=\text { Buoyancy launch angle frequency squared }
\end{aligned}
$$

where $\theta^{\prime}=$ internal gravity wave launch angle (relative to the horizontal); $H_{p}=$ pressure scale height (as earlier defined).

These resonant frequencies define the wave regimes in two branches (with evanescent Lamb waves existing at all frequencies, but exclusively between $\omega_{g}$ and $\left.\omega_{\mathrm{co}}\right)$ :

$\omega_{\text {co }} \leq \omega \leq \infty$ : Acoustics/infrasonic waves; $\omega_{\text {co }} \leq \omega \leq \omega_{g}$ : Internal gravity waves

The interested reader is referred to Mihalas and Weibull-Mihalas (1999) for an evaluation of these frequencies in hydrostatic, non-isothermal and ionized media respectively.

\subsubsection{Modeling approaches for $A G W$ 's}

The types of modeling approaches utilized for propagation of AGW's include "ray" or wave normal theory (geometrical acoustics), Normal mode waveguide (full wave) theory, Ray-mode theory and also numerical integration techniques, etc.

The wave normal "ray" tracing equations or Geometrical "particle" acoustics (non-dissipative limit) can be justified by using the size parameter, $S$, as defined in optics. If we define $S=2 \pi \cdot\{r / \lambda\}$, where $r=$ "obstacle scale" redirecting the wave and $\lambda=$ wavelength (at the maximum amplitude of the wave), then we can identify regimes as (a) $S \gg 1$, Geometrical acoustics, (b) $S \sim \mathrm{O}(1)$ : Wave diffraction regime or (c) $S \ll 1$ : Wave scattering regime

Furthermore, we can also define the ray mode transition distance for a uniform waveguide, i.e., $R_{\mathrm{rm}}=2 \cdot H^{2} / \lambda$ (Ceplecha et al., 1998), where $H=$ vertical duct thickness, then compare the current range, $R$ so that if:

(a) $R<R_{\mathrm{rm}}$, Geometrical acoustics is applicable (more "rays" than modes exist), whereas if

(b) $R>R_{\mathrm{rm}}$, Full wave theory is applicable (more modes than "rays" exist).

There are two geometric acoustics kinematic invariants in a horizontally stratified, steady, range-independent medium (Lindsay, 1960), namely: 
(a) Wave normal heading angle, $\phi$, as defined at the source: $\phi=$ constant

(b) Characteristic velocity (the Snell's law constant), $K=$ constant

For stationary point sources (for all possible azimuths):

$K(z)=\left(c_{s} / \cos \theta^{\prime}\right)$

$\theta^{\prime}=$ wave normal launch angle with respect to the local horizontal.

For moving line sources:

If $V(z) \gg c_{s}:$ Hypersonic flow

$K(z)=\left(c_{s} / \sin \theta\right) \cdot\left\{\sin ^{2} \theta+(1-2 \cdot(\Delta \phi / \pi))^{2} \cdot \cos ^{2} \theta\right\}^{1 / 2}$

$\theta=$ horizontal entry angle of the bolide.

For $V(z)>c_{s}$ : Supersonic flow or for supersonically moving point sources:

$$
K(z)=c_{s}(z) \cdot V(z) /\left\{\left|\left(V^{2}(z)-c_{s}^{2}(z)\right)^{1 / 2} \cdot \sin \theta-c_{s}(z) \cdot \cos \theta\right|\right\}
$$

The above treatment neglects non-linear refraction within $R_{0}$ of the trajectory.

If steady state winds are included the term, $\left|V_{H}\right| \cdot \cos (\phi-\psi(z))$ must also be added to the right hand side of the various expressions for $K(z)$.

\subsubsection{Wave energy conservation properties}

Wave kinetic energy density conservation: (Dissipationless wave propagation)

Kinetic energy density $\equiv 1 / 2 \cdot \rho(z) \cdot\left\{\Delta u^{2}(z)\right\}=$ constant since:

$\Delta u(z) \equiv \Delta p(z) /\left\{\rho(z) \cdot c_{s}(z)\right\}$ for acoustic (infrasonic) waves

$\Delta u(z)=$ perturbation wind due to the wave

$$
\therefore 1 / 2 \cdot \Delta p^{2}(z) /\left\{\rho(z) \cdot c_{s}^{2}(z)\right\}=\text { propagation constant }
$$

where $\rho(z)=\rho_{0} \cdot \exp \left(-z / H_{p}\right)$ in an isothermal, hydrostatic atmosphere for example; $\rho_{0}=$ surface air density

Thus, for upward (downward) propagation, we expect increasing (decreasing) effects of non-linearity and $\delta u(\mathrm{z})$ increases (decreases) exponentially while $\Delta p(z)$ decreases (increases) exponentially. Knowledge of the wave kinetic energy density at all points on the entry trajectory and of the infrasonic amplitude, $\Delta p$ at the ground $(z=0)$ allows a reliable calculation of the source energy (ReVelle et al., 2004). In general, the pressure wave amplitude of the propagating wave is expected to be a function of range, blast 
wave radius, line source length, differential acoustic efficiency, etc (Edwards et al., 2004).

\subsection{Documented IGW'S From BOLIDES}

The primary analytical theoretical treatment of AGW's from bolides is given by Golitsyn et al. (1977) for the far-field limit of "linearized" perturbations. A recent application of their asymptotic high frequency infrasonic technique can be found in Shumilov et al. (2003) for the Vitim bolide (see below). The application of infrasonic formulae in this case seems to be incorrect however since these observations are for the internal gravity waves from this bolide due to the very long observed periods.

The treatment in Golitsyn et al. although mathematically quite rigorous, suffers from the fact that it is not numerical or more flexible and can not be directly connected to the complex atmospheric environment through which AGW's must travel to a distant observer.

There have been a number of detections of IGW's from bolides, including Tunguska (6/30/1908), Revelstoke (3/31/1965) over Canada, possibly Kincardine (9/17/1966) over Lake Huron, other large bolides listed in ReVelle (1997), possibly the Crete bolide (6/02/2002) and finally the Vitim bolide (9/24/2002) over northwestern Siberia (Personal communication with O. Popova, Institute of Dynamics of Geospheres, Moscow). This topic has been studied very little compared to the infrasonic case, but it seems clear that the orientation of the bolide source with respect to the gravitational field of the earth is very important for IGW production. All of the above cases had rather flat entry angles with respect to the local horizon.

An analysis of the limiting cases of vertical and horizontal entry show that IGW's have their "parcel" oscillations parallel to the earth's gravitational field if the entry angle is close to being horizontal, but this is only the case for "rays" emanating at large azimuths outside of the plane of entry (nearly horizontal wave normals). For vertical entry, IGW's have their "parcel" oscillations parallel to the gravitational field. In such cases the gravitational field can provide a buoyant restoring force, thus efficiently producing IGW. Since the acoustical waves are longitudinal rather than transverse, these conclusions do not apply to them as expected.

In addition, Golitsyn et al. (1977) have also determined the differential conversion efficiency (relative to a point source) for AGW's from bolides:

$$
\eta_{\text {inf }}=(\gamma-1)^{2} /\left\{2(2 \pi)^{3 / 2}\right\} \approx 0.50 \%=\text { Differential acoustic efficiency }
$$




$$
\eta_{\operatorname{grv}}=(\gamma-1) /\left\{2(2 \pi)^{3 / 2}\right\} \approx 1.0 \%=\text { Differential IGW efficiency }
$$

with $\gamma=1.40$ for air considered as a perfect, diatomic gas (for Earth's atmosphere).

These above values can be readily compared to those computed using our most recent detailed entry modeling techniques (ReVelle, 2001a, 2002a, 2004):

(i) Neuschwanstein: $\sim 5.0 \%$ differential "acoustic" efficiency near the end of the visible trajectory.

(ii) Tagish Lake: $\sim 0.30 \%$ differential "acoustic" efficiency near the end of the visible trajectory.

(iii) Tunguska: $\sim 0.030 \%$ differential "acoustic" efficiency near the end of the visible trajectory.

Thus, there is a definite downward trend in $\varepsilon$ as size or mass increases. This makes good physical sense since $\varepsilon \propto\left\{1 / R_{0}^{2} \cdot l\right\}$ from our current theory.

3.4. WAVE NORMALS AND RAY PATHS: TRACING THE ATMOSPHERIC TRAJECTORIES OF INFRASONIC WAVES

The equations needed to describe the propagation paths of "linearized" AGW's in a horizontally stratified, range independent, steady state atmosphere can be written in the group velocity $\{x, y, z\}$ component form (Lindsay, 1960, ReVelle, 1976, Landau and Lifschitz, 1987):

$$
\begin{aligned}
& c_{g x}(z)=d x / d t=c_{s}(z) \cdot\{\alpha \cdot \sin \phi-\beta \cdot \cos \phi\}+u(z)+d \phi / d t \cdot y \\
& c_{g y}(z)=d y / d t=c_{s}(z) \cdot\{\alpha \cdot \cos \phi+\beta \cdot \sin \phi\}+v(z)-d \phi / d t \cdot x \\
& c_{g z}(z)=d z / d t= \pm c_{s}(z) \cdot \gamma+w(z)
\end{aligned}
$$

where $c_{s}^{2}=\{\gamma \cdot p / \rho\} ; c_{s}=f \cdot \lambda ; f=$ wave frequency; $\lambda=$ wavelength; $c_{s}=$ adiabatic thermodynamic phase velocity; $\{u, v, w\}=$ zonal, meridional and vertical wind components (time- and space-averaged values).

Equations (27a-27c) have direction cosines: $\alpha=\cos \theta ; \gamma=\sin \theta$; $\alpha^{2}+\beta^{2}+\gamma^{2}=1$ so that $\beta=0$ for a plane wave system. In this system of equations, locally plane waves were assumed with the wave propagation 
angle $\theta$ measured upward from the local horizontal and where $\phi=$ wave normal heading angle (measured clockwise from geographic North).

Throughout this description we are implicitly ignoring non-linear refractive effects due to the fact the energy deposition process modifies the medium locally so that the sound speed experiences a gradient along the wavefront with progressively lower temperatures farther away from the entry trajectory. This non-linear refraction occurs primarily within one blast wave relaxation radius of the trajectory $\left(x=1\right.$, where $\left.x=R / R_{0}\right)$ whereas all subsequent wave normal path tracing will be done for $x>10$.

Strictly speaking, the $d \phi / d t$ terms above are all zero in a range independent medium, but we have included them for completeness. We have also included $\beta$ terms for non-plane waves. Integration of these equations in a specified medium allows the resulting wave normal paths to be identified. The paths of these "wave normals" (not the corresponding "rays") are Galilean invariant and are the proper quantities to be evaluated (Hayes, 1971). Note that in a windless medium the ray and wave normal definitions are totally equivalent.

The wave normal paths can be readily identified if we assume an instantaneous source (so that a matching of the wavefront phase with its source altitude can be made) and the type of explosion event, i.e., a moving point versus a line source form of $K(z)$, etc. The basic difference between the two extreme limits of the characteristic velocity is that the infinite speed line sources are very directional unlike the stationary point source problem in which all "ray" launch directions are possible.

As discussed in Revelle $(1976,1997)$, the launched wave normals must satisfy the waveguide conditions in order for long distance ducting of the signal to occur, i.e., $K>c_{\text {eff }}(z=0)$ between the ground and various layers aloft in the Earth's atmosphere (Ceplecha et al., 1998). Examples of wave normal paths in three different planes, namely $\{x, z\},\{y, z\}$ and $\{x, y\}$ are given in detail for the Neuschwanstein bolide in ReVelle et al. (2004).

\section{Summary and Conclusions}

A theoretical entry model that includes the shape change parameter, $\mu$, that encompasses all major physical processes during entry has been developed and applied to a large range of meteoroid types and sizes. The model was developed utilizing an energetics analysis that successfully predicts the end height after a fixed percentage of the original kinetic energy has been exceeded. This approach was shown herein to be equivalent mathematically to the classical end height of ReVelle (1979). The treatment explicitly allows for either a homogeneous or a porous meteoroid and for either a single-body or a fragmentation cascade model (only triggered if the stagnation pressure on the 
frontal cross-section exceeded the breaking strength of the meteoroids). Fragments were transferred to the near-wake where they were either allowed to interact with the main leading body (collective wake model) or remained in the wake as they ablated away with time (non-collective wake model). An intermittent mode which invoked both of these limits is also being actively studied. Bolide luminosity was also computed for all cases using the recently developed, panchromatic luminous efficiency of ReVelle and Ceplecha (2001f, 2002). Available bolide luminosity outputs were properly calibrated in terms of either panchromatic stellar magnitude or in terms of Watts/ steradian (applicable at $100 \mathrm{~km}$ in the zenith).

Also, we performed a normalized panchromatic total power balance using the various differential efficiencies. We also developed a "first principles" form of the differential acoustical efficiency which was allowed to evolve independently of all of the other types which were functionally related to the panchromatic differential luminous efficiency. This approach has been applied to a number of cases including Neuschwanstein (ReVelle et al., 2004), etc. Finally, we compared these predictions to Golitsyn et al. (1977) and found some similarities, but also many differences.

Finally the topic of the spectrum of atmospheric AGW's has been presented in order to asses how this new channel of information, i.e., IGW's, can be used to assess bolide source properties. In addition the acoustical wave normal group velocity equations were presented for a horizontally stratified steady state range-independent atmosphere to study the complex refraction effects associated with bolide infrasound generation and ground-based detection. We have also summarized the energetic constraints during acoustic/infrasonic wave propagation that have also been successfully used to evaluate the Neuschwanstein meteorite fall (ReVelle et al., 2004).

There is much more future work yet to be done. This includes many more details on the fragmentation and related processes, high altitude transitional Knudsen number interference heating effects, precursor ionization/free stream absorption modeling, i.e., the radar head echo problem, temperature calculations for realistic conditions, etc. The latter calculations, for example, have already been formally carried out for the case of the leading shock front for the case of equilibrium, chemically reacting air using standard hypersonic aerodynamic methods, but there was not sufficient space to elaborate on this topic in this brief review.

\section{Appendix A: Energetics End Height Single-body Equation Equivalence}

$$
z_{\mathrm{KE}}(V)=-H_{p} \cdot\left\{\ln \left(p_{\infty}^{*} / p_{0}\right) \cdot \exp \left[-(\sigma \cdot F) \cdot V_{\infty}^{2}\right] \cdot\left\{D-D^{\prime}\right\}+\exp \left[-z^{\prime} / H_{p}\right]\right\}
$$




$$
\begin{aligned}
z^{\prime}= & -H_{p} \cdot\left\{\ln \left(p_{\infty}^{*} / p_{0}\right) \cdot\left(2 g H_{p} / V_{\infty}^{2}\right)\right\} \\
D^{\prime}= & -\left\{E i\left[(\sigma \cdot F) \cdot V_{\infty}^{2}\right]-E i\left[(\sigma \cdot F) \cdot V^{2}(z)\right]\right. \\
& \left.-\ln \left(V_{\infty} / V(z)\right)^{2}-\left[(\sigma / 2)\left(V_{\infty}^{2}-V^{2}(z)\right)\right]\right\}
\end{aligned}
$$

where $\operatorname{Ei}(\sigma \cdot F)=$ the exponential integral function; $F=(1-\mu) / 2 ;-2\left({ }^{*}\right)$ $\leq \mu \leq \sim 1 ; \mu=2 / 3$ is the self-similar value (no shape change); $p_{\infty}{ }^{*}=\mathrm{mg} \cdot \sin \theta /$ $\left(C_{D} A\right)=$ modified ballistic entry parameter; $p_{\infty}^{*}=4 \cdot \rho_{m} \cdot r \cdot g \cdot \sin \theta /\left(3 C_{D}\right)$ for a sphere; $p_{0}=$ surface pressure; $D=4.605$ for $99 \%$ kinetic energy depletion at the end height.

\section{(*): Effective "pancake" model limits for $\mu<0$}

Starting from the classical (geopotential) end height equation using standard notation as given in ReVelle $(1979,1980,1987)$ written assuming $\mu=2 / 3$ so that $F=(1-\mu) / 2=1 / 6$ for simplicity with $\sigma=$ constant, i.e., the so-called simple ablation theory:

$$
\begin{aligned}
& z(V)=-H_{p} \cdot \ln \left\{\exp \left(-z^{\prime} / H_{p}\right)+2\left(p_{\infty}^{*} / p_{0}\right) \cdot \exp \left[-\sigma V_{\infty}^{2} / 6\right] \cdot(\Delta E i / 2)\right\} \\
& \mathrm{KE}(z)=\mathrm{KE}_{\infty} \cdot \exp [-D] \\
& M(z)=M_{\infty} \cdot \exp \left[-(\sigma / 2) \cdot\left\{V_{\infty}^{2}-V^{2}(z)\right\}\right]
\end{aligned}
$$

where

$$
\begin{aligned}
& \Delta E i \equiv E i\left(\sigma V_{\infty}^{2} / 6\right)-E i\left(\sigma \cdot V(z)^{2} / 6\right) \\
& \mathrm{KE}=1 / 2 m \cdot V^{2} ; \mathrm{KE}=1 / 2 m_{\infty} \cdot V_{\infty}^{2} ; D=\alpha+\beta(\text { ReVelle, } 1980)
\end{aligned}
$$

As shown in ReVelle (1980), $\Delta E i$ can be expanded in an infinite length power series form that can also be expressed as the difference between two very simple functions in (A.9):

$$
\begin{aligned}
\Delta E i= & \ln \left(\left\{\sigma V_{\infty}^{2} / 6\right\} /\left\{\sigma V^{2}(z) / 6\right\}\right)+\Delta=\ln \left(V_{\infty}^{2} / V^{2}(z)\right)+\Delta \\
\Delta= & (\sigma / 6) \cdot\left(V_{\infty}^{2}-V^{2}(z)\right)+(1 / 4) \cdot(\sigma / 6)^{2} \cdot\left(V_{\infty}^{4}-V^{4}(z)\right) \\
& +(1 / 18) \cdot(\sigma / 6)^{3} \cdot\left(V_{\infty}^{6}-V^{6}(z)\right)+\cdots
\end{aligned}
$$




$$
\Delta=\Delta E i-\ln \left(V_{\infty}^{2} / V^{2}(z)\right)
$$

Also, from (A.5) and (A.6) we can write:

$$
\begin{aligned}
& V(z)=V_{\infty}\left(M_{\infty} / M(z)\right)^{1 / 2} \cdot \exp [-D / 2] \\
& \left(M_{\infty} / M(z)\right)^{1 / 2}=\exp \left[(\sigma / 4) \cdot\left(V_{\infty}^{2}-V^{2}(z)\right)\right]
\end{aligned}
$$

Combining these two expressions in (A.10) and (A.11), we can also write:

$$
\ln \left(V(z) / V_{\infty}\right)=(\sigma / 4) \cdot\left(V_{\infty}^{2}-V^{2}(z)\right)-D / 2
$$

Rearranging (A.4) into a form solved for the velocity and as the natural logarithm of the velocity as a function of $z$, we also have:

$$
\begin{aligned}
V(z)=V_{\infty} \cdot \exp \left\{-\left[p_{0} /\left(2 \cdot p_{\infty}^{*}\right)\right\} \cdot \exp \left[\sigma \cdot V_{\infty}^{2} / 6\right] \cdot\left(\exp \left(-z / H_{p}\right)\right.\right. \\
\left.-\exp \left(-z^{\prime} / H_{p}\right)-\Delta / 2\right] \\
\begin{aligned}
\ln \left(V(z) / V_{\infty}\right)= & -\left[p_{0} /\left(2 \cdot p_{\infty}^{*}\right) \cdot \exp \left[\sigma \cdot V_{\infty}^{2} / 6\right] \cdot\left(\exp \left(-z / H_{p}\right)\right.\right. \\
& \left.-\exp \left(-z^{\prime} / H_{p}\right)-\Delta / 2\right]
\end{aligned}
\end{aligned}
$$

Equating (A.12) and (A.14), we can solve again for $z(V)$ in the form:

$$
\begin{aligned}
z(V)= & -H_{p} \cdot\left\{\operatorname { l n } \left[\left\{p_{\infty}^{*} / p_{0}\right\} \cdot \exp \left[-\sigma V_{\infty}^{2} / 6\right] .\right.\right. \\
& \left.\left.\left\{D-(\sigma / 2) \cdot\left(V_{\infty}^{2}-V^{2}(z)\right)+\Delta\right\}+\exp \left(-z^{\prime} / H_{p}\right)\right]\right\}
\end{aligned}
$$

Defining:

$$
\begin{aligned}
& D^{\prime} \equiv(\sigma / 2) \cdot\left(V_{\infty}^{2}-V^{2}(z)\right)-\Delta \\
& z(V)=-H_{p} \cdot \ln \left[\left\{p_{\infty} * / p_{0}\right\} \cdot \exp \left[-\sigma V_{\infty}^{2} / 6\right] \cdot\left\{D-D^{\prime}\right)\right\}+\exp \left(-z^{\prime} / H_{p}\right)
\end{aligned}
$$

where

$$
D^{\prime}=-\left\{\Delta E i-\ln \left(V_{\infty} / V(z)\right)^{2}-(\sigma / 2) \cdot\left(V_{\infty}^{2}-V^{2}(z)\right\}\right.
$$

Equation (A.17) and (A.18) are the desired results. Thus the classical end height equation has been shown to be totally equivalent to the energetics approach used throughout this paper written in terms of the $D$ parameter of 
ReVelle. The solutions that we have used to make predictions use the variable ablation parameter form of these equations developed in ReVelle (1979). Thus, all solutions are numerical results in thin vertical layers over small velocity change intervals rather than the simple analytic results developed above.

\section{Acknowledgements}

I would like to thank the ISR-DR Program Office at Los Alamos National Laboratory for their continuous support throughout the course of this work, especially, Mr. Mark Hodgson. I would also like to thank DOE HQ in NA22 for their continuing support as well. Finally, I would like to dedicate this paper to the memory of my beloved father, Mark A. Revelle.

\section{References}

Baldwin, B. and Sheaffer, Y.: 1971, J. Geophys. Res. 76, 4653-4668.

Beech, M. and Brown, P.: 2000, Planet. Space Sci. 48, 925-932.

Borovicka, J. and Spurny, P.: 1996, Icarus 121, 484-510.

Borovicka, J., Popova, O. P., Nemtchinov, I. V., Spurny, P., and Ceplecha, Z.: 1998a, Astron. Astrophys. 334, 713-728.

Borovicka, J., Popova, O. P., Golub, A. P., Kosarev, I. B., and Nemtchinov, I. V.: 1998b, Astron. Astrophys. 337, 591-602.

Brown, P. G., ReVelle, D. O., Tagliaferri, E., and Hildebrand, A. R.: 2002, Meteoritics Planet. Sci. 37, 661-675.

Ceplecha, Z., Borovicka, J., Elford, W. G., ReVelle, D. O., Hawkes, R. L., Porubcan, V., and Simek, M.: 1998, Space Sci. Rev. 84, 327-471.

Ceplecha, Z. and ReVelle, D. O.: 2005, Meteoritics Planet. Sci. 40, 35-54.

Edwards, W. N., Brown, P. G. and ReVelle, D. O.: 2004, Earth, Moon, Planets, (this issue).

Golitsyn, G. S., Grigor'yev, G. I., and Dokuchayev, V. P.: 1977, Atmos. Oceanic Phys. 13, 633-639 English Translation.

Greenhow, J. S. and Hawkins, G. S.: 1952, Nature 170, 355-357.

Hayes, W. D.: 1971, in VanDyke, M., Vincenti, W. G., and Wehausen, T. V. (eds.), Sonic Boom, Annual Review of Fluid Mechanics, Annual Review Inc. vol. 3, Palo Alto CA, pp. 269-290.

Jones, D. L., Goyer, G. G., and Plooster, M. N.: 1968, J. Geophys. Res. 73, 3121-3127.

Kraemer, D. R. and Bartman, F. L.: 1981, in Proceedings of the International Symposium on Acoustic Remote Sensing of the Atmosphere and the Oceans, Chapter V., University of Calgary Press, Calgary, Alberta, Canada, pp. 31-49.

Landau, L. D. and Lifschitz, E. M.: 1987. Fluid Mechanics (2nd ed.). Pergamon Press, Oxford, $539 \mathrm{pp}$.

Lin, S. C.: 1954, J. Appl. Phys. 25, 54-57.

Lindsay, R. B.: 1960, Mechanical Radiation, McGraw- Hill Book Company, New York, 415 pp.

Mihalas, D. and Weibel-Mihalas, B.: 1999, Foundations of Radiation Hydrodynamics, Dover Publications Inc., Mineola New York, 718 pp.

Opik, E. J.: 1958, Physics of Meteor Flight in the Atmosphere, Interscience Publishers Inc., New York, 174 pp. 
Plooster, M. N.: 1968. Shock Waves from Line Sources, National Center for Atmospheric Research (NCAR), Boulder, CO, NCAR Technical Note TN-37.

Plooster, M. N.: 1971, Phys. Fluids 13, 2665-2675.

ReVelle, D. O.: 1976, J. Geophys. Res. 81, 1217-1230.

ReVelle, D. O.: 1979, J. Atmos. Terr. Phys. 41, 453-473.

ReVelle, D. O.: 1980, Geophys. Res. 85, 1803-1808.

ReVelle, D. O.: 1983, Meteoritics 18, 386.

ReVelle, D. O.: 1987, The End Height of Fireballs as a function of their Residual Kinetic Energy, Handbook for MAP (Middle Atmosphere Program), Volume 25, Proceedings of the First GLOBMET Symposium, Dushanbe, Tajicistan, USSR, August 19-24, 1985, SCOSTEP Secretariot, University of Illinois, Urbana, editor, R.G. Roper, August, 1987, pp. 255-257.

ReVelle, D. O.: 1993, in Stohl, J. and Williams, I. P. (eds.), Meteoroids and their Parent Bodies, Astronomical Institute of the Slovak Academy of Sciences , Bratislava, Slovakia, pp. 343346.

ReVelle, D. O.: 1997, Historical Detection of Atmospheric Impacts of Large Bolides Using Acoustic-Gravity Waves, Annals of the New York Academy of Sciences, Near-Earth Objects - The United Nations International Conference, editor, J.L. Remo, The New York Academy of Sciences, New York, New York, 822, pp. 284-302.

ReVelle, D. O. and Whitaker, R. W.: 1999, Meteoritics Planet. Sci. 34, 995-1005.

ReVelle, D. O.: 2001a, Theoretical Leonid Entry Modeling, Proceedings Meteoroids2001 Conference, 6-10 August 2001, Swedish Institute of Space Physics, Kiruna, Sweden, ESA SP-495, ESTEC, Noordwijk, The Netherlands, B. Warmbein editor, November, 2001, pp. 149-154.

ReVelle, D. O.: 2001b, Large Leonid Entry Modeling: Application to the Bolide of 11/17/ 1998, Proceedings Meteoroids2001 Conference, 6-10 August 2001, Swedish Institute of Space Physics, Kiruna, Sweden, ESA SP-495, ESTEC, Noordwijk, The Netherlands, B. Warmbein editor, November, 2001, pp. 179-184.

ReVelle, D. O.: 2001c, Bolide Fragmentation Processes: Single-Body Modeling versus the Catastrophic Fragmentation Limit, Proceedings Meteoroids2001 Conference, 6-10 August 2001, Swedish Institute of Space Physics, Kiruna, Sweden, ESA SP-495, ESTEC, Noordwijk, The Netherlands, B. Warmbein editor, November, 2001, pp. 491-496.

ReVelle, D. O.: 2001d, Global Infrasonic Monitoring of large Bolides, Proceedings Meteoroids2001 Conference, 6-10 August 2001, Swedish Institute of Space Physics, Kiruna, Sweden, ESA SP-495, ESTEC, Noordwijk, The Netherlands, B. Warmbein editor, November, 2001, pp. 483-490.

ReVelle, D. O.: 2001e, Bolide Dynamics and Luminosity Modeling: Comparisons between Uniform Bulk Density and Porous Meteoroids Models, Proceedings Meteoroids2001 Conference, 6-10 August 2001, Swedish Institute of Space Physics, Kiruna, Sweden, ESA SP-495, ESTEC, Noordwijk, The Netherlands, B. Warmbein editor, November, 2001, pp. 513-518.

ReVelle, D. O. and Ceplecha, Z.: 2001f, Bolide Physical Theory with Application to PN and EN Fireballs, Proceedings Meteoroids2001 Conference, 6-10 August 2001, Swedish Institute of Space Physics, Kiruna, Sweden, ESA SP-495, ESTEC, Noordwijk, The Netherlands, B. Warmbein editor, November, 2001, pp. 507-512.

ReVelle, D. O. and Ceplecha, Z.: 2001g, Calculations of Shape Change and Fragmentation Parameters Using very Precise Bolide Data, Proceedings Meteoroids2001 Conference, 610 August 2001, Swedish Institute of Space Physics, Kiruna, Sweden, ESA SP-495, ESTEC, Noordwijk, The Netherlands, B. Warmbein editor, November, 2001, pp. 551-556. 
ReVelle, D. O.: 2002a, Fireball Dynamics, Energetics, Ablation, Luminosity and Fragmentation Modeling, Proceedings of Asteroids, Comets, Meteors ACM 2002, 29 July-2 August 2002, Technical University Berlin, Berlin, Germany, ESA SP-500, ESTEC, Noordwijk, The Netherlands, B. Warmbein editor, November, 2002, pp. 127-136.

ReVelle, D. O.: 2002b, Porosity: A Natural Alternative Explanation of Bolide Types, their Atmospheric Behavior and the Implications, Proceedings of Asteroids, Comets, Meteors ACM 2002, 29 July-2 August 2002, Technical University Berlin, Berlin, Germany, ESA SP-500, ESTEC, Noordwijk, The Netherlands, B. Warmbein editor, November, 2002, pp. 233-236.

ReVelle, D. O. and Ceplecha, Z. 2002, Semi-empirical Fragmentation Model of Meteoroid Motion and Radiation during Atmospheric Penetration, Proceedings of Asteroids, Comets, Meteors ACM 2002, 29 July-2 August 2002, Technical University Berlin, Berlin, Germany, ESA SP-500, ESTEC, Noordwijk, The Netherlands, B. Warmbein editor, November, 2002, pp. 285-288.

ReVelle, D. O., Brown, P. G., and Spurny, P.: 2004, Meteoritics Planet. Sci. 39, 1605-1626.

Romig, M. F.: 1965, Am. Inst. Aeronaut. Astronaut. J. 3, 385-394.

Sepri, P., Chen, K. K., and O'Keefe, J. A.: 1981, J. Geophys. Res. 86, 5103-5111.

Shumilov, O. I., Kasatkina, E. A., Tereshchecnko, E. D., Kulichkov, S. N., and Vasil'ev, A. N.: 2003, JETP Lett. 77, 115-117.

Spurny, P., Spalding, R. E. and Jacobs, C.: 2001, Common Ground-Based Optical and Radiometric Detections within the Czech Fireball Network, Proceedings Meteoroids2001 Conference, 6-10 August 2001, Swedish Institute of Space Physics, Kiruna, Sweden, ESA SP-495, ESTEC, Noordwijk, The Netherlands, B. Warmbein editor, November, 2001, pp. $135-140$. 